\title{
A Multi-scale 3D Otsu Thresholding Algorithm for Medical Image
}

\section{Segmentation}

\author{
Xiaoli Zhang ${ }^{1,2}$, Haiying Zhao ${ }^{3}$, Xiongfei $\mathrm{Li}^{1,2, *}$, Yuncong Feng ${ }^{1,2}$, Hongpeng $\mathrm{Li}^{4}$ \\ ${ }^{1}$ Key Laboratory of Symbolic Computation and Knowledge Engineering of Ministry of Education, Jilin \\ University, Changchun 130012, China \\ ${ }^{2}$ College of Computer Science and Technology, Jilin University, Changchun 130012, China \\ ${ }^{3}$ Mobile Media and Cultural Calculation Key Laboratory of Beijing, Century College, BUPT, Beijing 102101, \\ China \\ ${ }^{4}$ Department of Radiology, The Second Hospital of Jilin University, Changchun 130041, China
}

\begin{abstract}
Thresholding technique is one of the most imperative practices to accomplish image segmentation. In this paper, a novel thresholding algorithm based on 3D Otsu and multi-scale image representation is proposed for medical image segmentation. Considering the high time complexity of 3D Otsu algorithm, an acceleration variant is invented using dimension decomposition rule. In order to reduce the effects of noises and weak edges, multi-scale image representation is brought into the segmentation algorithm. The whole segmentation algorithm is designed as an iteration procedure. In each iteration, the image is segmented by the efficient 3D Otsu, and then it is filtered by a fast local Laplacian filtering to get a smoothed image which will be input into the next iteration. Finally, the segmentation results are pooled to get a final segmentation using majority voting rules. The attractive features of the algorithm are that its segmentation results are stable, it is robust to noises and it holds for both bi-level and multi-level thresholding cases. Experiments on medical MR brain images are conducted to demonstrate the effectiveness of the proposed method. The experimental results indicate that the proposed algorithm is superior to the other multilevel thresholding algorithms consistently.
\end{abstract}

Keywords: Image segmentation; 3D Otsu thresholding; Multi-scale image representation; Local Laplacian filtering

\section{Introduction}

Image segmentation refers to a technology capable of partitioning an image into non-overlapping objects of interest based on the intrinsic features, such as intensity, texture, color and contrast $[1,2]$. Nowadays, image segmentation technique has been applied to various images: gray or color natural images [3-5], topographic maps [6], satellite images [7, 8], and medical images [9, 10].

In medical filed, image segmentation can be used for assisting diagnosing tumors in brain or any part of the body from the MRI scan or PET scan [11]. Segmentation of the human cerebral cortex has

* Corresponding author

Email: xiaolizhang1987@gmail.com (Xiaoli Zhang), zhaohaiying@bupt.edu.cn (Haiying Zhao), 1xf@jlu.edu.cn (Xiongfei Li), fengyc14@ mails.jlu.edu.cn (Yuncong Feng), hongpengli1991@126.com (Hongpeng Li). 
been extensively studied [12, 13].Until now, a wide variety of segmentation algorithms has been proposed in the last decades $[14,15]$. Generally, these algorithms can be divided into four categories: (1) thresholding [16-18], (2) clustering-based [19], (3) levelset-based [20] and (4) graphcut-based [21]. Among them, the histogram-based thresholding is commonly regarded as a very popular technique owing to its simplicity and efficiency. The most classical image thresholding approaches include maximum between-class variance (Otsu) method [22], minimum error method [23] and maximum entropy method [24]. In addition, literatures [25-28] studied real time applications of the Otsu method. In 2012, Lin et al. proposed an improved Tsallis entropy and local long-range correlation based thresholding algorithm for image segmentation [29]. Hongmin Cai et al. presented an iterative tri-class thresholding technique based on Otsu method, in which the object region in an image is distinguished from the background region iteratively, rather than processing the full image as a whole region [30]. Long et al. proposed an adaptive 3D minimum error thresholding algorithm based on the 3D reconstruction and dimension reduction technique [31]. These thresholding algorithms can be easily extended to multilevel thresholding which is a critical and challenging work [7]. However, multilevel thresholding based image segmentation is inefficient in determining the optimal thresholds for the time complexity grows exponentially with the number of thresholds increasing [32].

Over the years, many improved thresholding techniques have been developed to solve the efficiency problem. Soham Sarkar et al. presented a novel multilevel thresholding method for unsupervised partition between objects and background from an image based on minimum cross entropy (MCE) [3]. In this method, differential evolution (DE) is employed to improve the computation time and robustness. AK Bhandari et al. proposed a new approach for color image segmentation using cuckoo search (CS) algorithm which is supported by the Tsallis entropy based multilevel thresholding [7]. S. Manikandan et al. developed a multilevel thresholding for medical image segmentation which utilizes a real coded genetic algorithm with simulated binary crossover (SBX) to maximize the entropy [16]. In paper [17], an adaptive bacterial foraging (ABF) algorithm is put forward for brain MRI segmentation, which employs an adaptive step size to improve the search ability of traditional bacterial foraging (BF) algorithm. Kamal Hammouche et al. offered an automatic multilevel thresholding method by combining a genetic algorithm with a wavelet transform, which can determine the proper number of thresholds as well as the optimal threshold values automatically [33].

Despite the critical research and key advances in the past years, several challenges still remain in the multilevel thresholding technique. Efficient, robust and automatic segmentation of medical images is one such challenge [34]. In this paper, we proposed a multi-scale 3D Otsu thresholding algorithm for medical image segmentation. 3D Otsu single thresholding is a ubiquitous method, in which both the pixel's spatial neighborhood information and pixel gray values are considered, so it possesses de-noising performance and can achieve acceptable segmentation results. However, 3D Otsu multilevel thresholding is seldom investigated, which may be due to its high computation cost. In our method, an efficient 3D Otsu scheme based on decomposition rule is designed for multilevel thresholding segmentation. Jin et al. proposed a vascular tree segmentation algorithm based on Hessian-based multiscale filtering. The experimental results show that multiscale image representation is conducive to get more precise segmentation [35]. In this paper, a fast local 
Laplacian filtering technique is employed to obtain multiple scales of the input image, and then 3D Otsu is performed on those images to get a series of segmentation maps. Finally, these segmentation maps can be combined to get the final segmentation result. The key contribution of the work can be summarized as follows,

(1) An efficient 3D Otsu segmentation algorithm is proposed based on dimension decomposition.

(2) Images are smoothed interactively using an edge-aware filter to reduce the influence of noises and weak edges.

(3) A rule for fusing segmentation maps is proposed to make the segmentation get the maximum between-class variance.

The rest of this paper is structured as follows: Section 2 describes the proposed multi-scale efficient 3D Otsu thresholding algorithm in detail; in Section 3, experimental results and analysis are presented; finally, conclusions are drawn in Section 4.

\section{The proposed segmentation algorithm}

In this section, the multi-scale 3D Otsu thresholding algorithm (MS3D Otsu) for medical image segmentation is presented in detail. The attractive features of this algorithm are that its segmentation results are stable, it is robust to noises and it holds for both bi-level and multi-level thresholding cases.

\subsection{Segmentation scheme}

Let $I$ denote an original image to be segmented. As displayed in Fig.1, the scheme of the proposed segmentation algorithm can be summarized as follows,

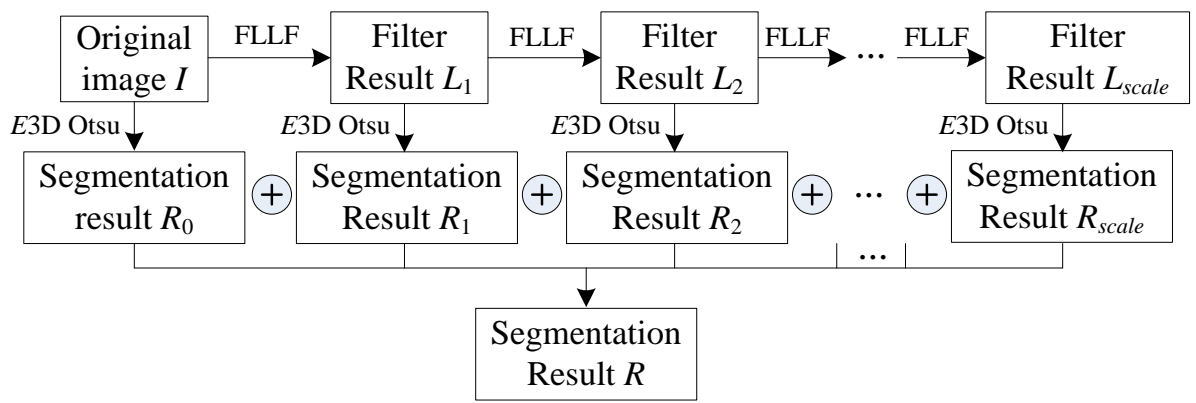

Fig.1 The framework of the proposed segmentation algorithm

Step.1 The original image $I$ is segmented by using a new efficient 3D Otsu thresholding method (E3D Otsu) which will be detailed in subsection 3.3, and the segmentation result is denoted as $R_{0}$.

Step.2 Perform the fast local Laplacian filtering (FLLF) on image $I$ to obtain the filtered image $L_{1}$; use E3D Otsu to segment $L_{1}$, and the segmentation result is represented as $R_{1}$. Run Step 2 iteratively: in the $(i+1)_{t h}$ iteration, $L_{i}$ is first filtered by FLLF to get a more smooth image $L_{i+1}$, and then segmentation result $R_{i+1}$ of $L_{i+1}$ is obtained by E3D Otsu. 
Step.3 After finishing the iteration, fuse all the segmentation results $\left\{R_{0}, R_{1}, R_{2}, \ldots, R_{\text {scale }}\right\}$ to obtain the final segmentation result $R$.

\subsection{Image multi-scale representation}

The aim of bringing the image multi-scale representation into segmentation algorithm is to remove the influence of noise, weak edges or textures on segmentation results. However, edges are greatly important to segmentation, hence a better image multi-scale representation should be edge-aware.

Until now, many image multi-scale representation algorithms have been invented. Laplacian pyramid is one of classical algorithms. Nevertheless, the Laplacian pyramid is blamed for incapability of representing edges well and ill-suited applications in edge-aware operations such as edge-preserving smoothing and tone mapping, because it is constructed with Gaussian kernels which are spatially invariant. As edges are of great significance to image segmentation, Laplacian pyramid does not hold for multi-scale image decomposition in image segmentation algorithm. In 2011, Sylvain Paris et al. invented a Local Laplacian filtering (LLF) algorithm which is a simple and flexible approach for edge-aware image processing based on the Laplacian pyramid [36]. In the algorithm, edges are first classified into two catalogues with a threshold: large-scale edges and small-scale details. Then a set of image filters are designed to achieve edge-preserving smoothing, detail enhancement, etc. However, these filters are not efficient enough.

For an input image $I$ with the size of $M \times N$, one level local Laplacian filtering is defined as

$$
L_{1, \mathbf{p}}=I_{\mathbf{p}}+\sum_{\mathbf{q} \in \Omega_{\mathbf{p}}} \bar{G}_{\sigma_{\mathbf{p}}}(\mathbf{p}-\mathbf{q}) f\left(I_{\mathbf{p}}-I_{\mathbf{q}}\right)\left(I_{\mathbf{p}}-I_{\mathbf{q}}\right)
$$

where $\mathbf{p}$ and $\mathbf{q}$ denote pixels' locations, hence $I_{\mathbf{p}}$ refers to the intensity of the original image $I$ at location p. $\Omega_{\mathbf{p}}$ denotes the neighborhood of pixel p. $\bar{G}_{\sigma_{\mathbf{p}}}=\frac{1}{\sqrt{2 \pi \sigma_{\mathbf{p}}^{2}}} G_{\sigma_{\mathbf{p}}}$ is a normalized Gaussian kernel and $G_{\sigma_{\mathrm{p}}}$ is a Gaussian kernel of $\sigma_{p}^{2}$ defined by $G_{\sigma_{p}}=e^{-x^{2} / 2 \sigma_{p}^{2}} . f$ is a continuous function. From Eq.(1), it can be noted that $\bar{G}_{\sigma_{\mathrm{p}}}$ can be regarded as spatial weight and $f$ be the range weight.

The time complexity of the local laplacian filter is $O(M \times N \times \# \Omega)$ (\# $\Omega$ denotes the size of neighborhood), hence it is not efficient enough. In [37], Aubry et al. argued that it is not necessary to compute $f\left(I_{\mathbf{p}}-I_{\mathbf{q}}\right)\left(I_{\mathbf{p}}-I_{\mathbf{q}}\right)$ for all the pixels in the neighborhood of pixel p. For the sake of discussion, $f\left(I_{\mathbf{p}}-I_{\mathbf{q}}\right)\left(I_{\mathbf{p}}-I_{\mathbf{q}}\right)$ is re-written as $d(i-g)$ where $i$ and $g$ denote $I_{\mathbf{p}}$ and $I_{\mathbf{q}}$, respectively. For different pixel $\mathbf{q} \in \Omega_{\mathbf{p}}, i$ is constant but $g$ is not. If $g$ were constant, we would need to compute $d$ only once per pixel. Then, the time complexity of the algorithm would be $O(M \times N)$. However, the quality of the algorithm will be badly affected. Mathieu Aubry et al. proposed to compute $d$ only for $\mathrm{K}(<<\# \Omega)$ values of $g$ and interpolate them. Then, the algorithm's time complexity is reduced to $O(M \times N \times K)$.

Fig. 2 gives an example of filtered results obtained by the fast local Laplacian filtering. Fig.3(a) is a source image and the corresponding filtered image is shown in Fig.3(b). By comparing the two 
images, it can be noted that the edges are represented well and brain tissues displayed as small-scale details are removed. In order to compare the two images' difference intuitively, we sampled a row of pixels at $y=300$ from subfigure (a) and (b) and show them in subfigure (c), which also justifies the appealing performance of the filter on edge preserving and detail removals.

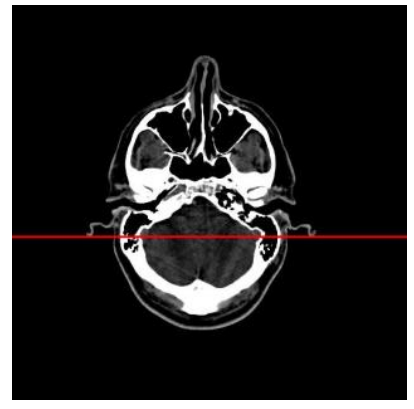

(a)

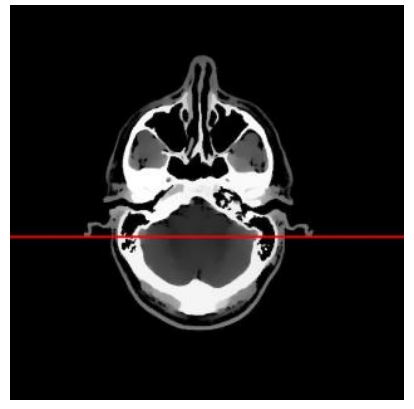

(b)

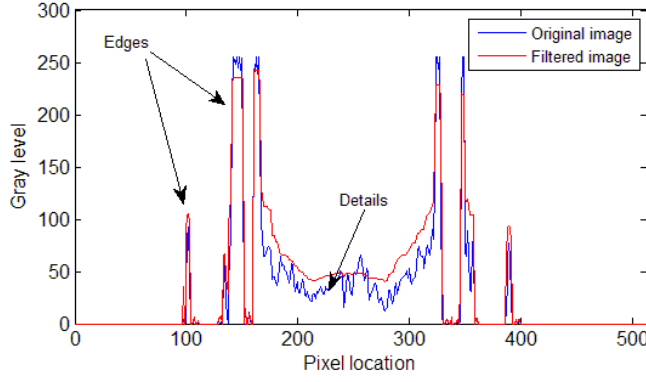

(c)

Fig.2 Illustration of filtered results obtained by the FLLF: (a) original image (size:512×512); (b) filtered image; (c) intensity plots along a sampled row of pixels at $y=300$ in subfigure (a) and (b).

\subsection{Efficient 3D Otsu thresholding}

Otsu is a ubiquitous thresholding algorithm in the field of image segmentation. 3D Otsu takes the pixel's spatial information including the neighborhood mean and median into account except the intensity, so it can improve segmentation results and has better noise immunity comparing to 1D Otsu and 2D Otsu. However, the time complexity of 3D Otsu is relatively high, especially for the case of multilevel thresholding, time complexity will exponentially grow as the number of thresholds increases. In [5], Jing et al. described a fast scheme for 3D Otsu which lowered the time complexity from $O\left(L^{6}\right)$ to $O\left(L^{3}\right)$ in the case of bi-level thresholding. But processing an image with the size of $256 * 256$ still takes 100 s, which severely limits 3D Otsu's practical applications. Therefore, we use the efficient variant of 3D Otsu that preserves its accuracy, while significantly reducing complexity [38].

In the efficient 3D Otsu method, we use 1D Otsu scheme to gain the optimal thresholds about pixels' gray value $f$, neighborhood mean $g$ and median $h$, respectively. As shown in Fig.3, the 3D Otsu histogram is constructed by taking $f, g$ and $h$ as x-axis, y-axis and z-axis. A point $(x, y, z)$ $(0 \leq x, y, z \leq L-1)$ in the 3D histogram represents a pixel in image $I$.

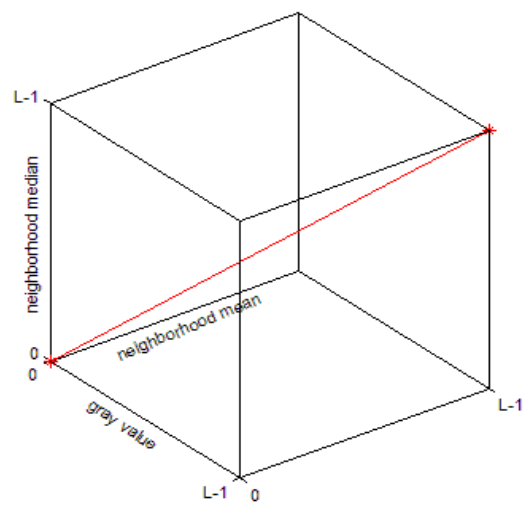

Fig.3 3D Otsu histogram 
For an original image $I$, its total number of pixels can be denoted as Num $=\sum_{x=0}^{L-1} n_{x}=\sum_{y=0}^{L-1} n_{y}=\sum_{z=0}^{L-1} n_{z}(0 \leq x, y, z \leq L-1)$, where $n_{x}$ refers to the number of pixels with the gray value $x, n_{y}$ denotes the number of pixels with the neighborhood mean $y$, and $n_{z}$ represents the number of pixels with the neighborhood median $z$. Then the probabilities of $n_{x}, n_{y}$ and $n_{z}$ are defined as $p_{x}^{(f)}=\frac{n_{x}}{N u m}, \quad p_{y}^{(g)}=\frac{n_{y}}{N u m} \quad$ and $p_{z}^{(h)}=\frac{n_{z}}{N u m}\left(p_{x}^{(f)}, p_{z}^{(h)}, p_{y}^{(g)} \geq 0\right)$, respectively.

We assume that image $I$ will be segmented into $K(K \geq 2)$ classes by $K-1$ groups of thresholds $\left\{\left(r_{1}, s_{1}, t_{1}\right), \ldots,\left(r_{K-1}, s_{K-1}, t_{K-1}\right)\right\}$, particularly " $K=2$ " refers to the case of single threshold segmentation. The between-class variances about the three elements (gray value $f$, neighborhood mean $g$ and median $h$ ) are defined as follows,

$$
\left\{\begin{array}{l}
\sigma_{B(f)}^{2}=\sum_{q=1}^{K} \omega_{q}^{(f)}\left(\mu_{q}^{(f)}-\mu_{T}^{(f)}\right)^{2} \\
\sigma_{B(g)}^{2}=\sum_{q=1}^{K} \omega_{q}^{(g)}\left(\mu_{q}^{(g)}-\mu_{T}^{(g)}\right)^{2} \\
\sigma_{B(h)}^{2}=\sum_{q=1}^{K} \omega_{q}^{(h)}\left(\mu_{q}^{(h)}-\mu_{T}^{(h)}\right)^{2}
\end{array}\right.
$$

where $\omega_{q}$ and $\mu_{q}$ denote the probability and mean of class $q(1 \leq q \leq K)$, respectively. They are given by

$$
\left\{\begin{array}{l}
\omega_{q}^{(f)}=\sum_{x=r_{q-1}}^{r_{q}} p_{x}^{(f)},\left(r_{0}=0\right) \\
\omega_{q}^{(g)}=\sum_{y=s_{q-1}}^{s_{q}} p_{y}^{(g)},\left(s_{0}=0\right), \\
\omega_{q}^{(h)}=\sum_{z=t_{q-1}}^{t_{q}} p_{z}^{(h)},\left(t_{0}=0\right)
\end{array}\right.
$$

and

$$
\left\{\begin{array}{l}
\mu_{q}^{(f)}=\sum_{x=r_{q-1}}^{r_{q}} \frac{x p_{x}^{(f)}}{\omega_{q}^{(f)}},\left(r_{0}=0\right) \\
\mu_{q}^{(g)}=\sum_{y=s_{q-1}}^{s_{q}} \frac{y p_{y}^{(g)}}{\omega_{q}^{(g)}},\left(s_{0}=0\right) \\
\mu_{q}^{(h)}=\sum_{z=t_{q-1}}^{t_{q}} \frac{z p_{z}^{(h)}}{\omega_{q}^{(h)}},\left(t_{0}=0\right)
\end{array}\right.
$$

where $\mu_{T}$ represents the total mean of all the classes, and it is defined as 


$$
\left\{\begin{array}{l}
\mu_{T}^{(f)}=\sum_{x=0}^{L-1} x p_{x}^{(f)} \\
\mu_{T}^{(g)}=\sum_{y=0}^{L-1} y p_{y}^{(g)} \\
\mu_{T}^{(h)}=\sum_{z=0}^{L-1} z p_{z}^{(h)}
\end{array} .\right.
$$

Our efficient 3D Otsu thresholding aims at finding the optimal thresholds $\left\{r_{1}^{*}, \ldots, t_{K-1}^{*}\right\}$, $\left\{s_{1}^{*}, \ldots, t_{K-1}^{*}\right\}$ and $\left\{t_{1}^{*}, \ldots, t_{K-1}^{*}\right\}$ to maximize the three objective functions $\sigma_{B(f)}^{2}, \sigma_{B(g)}^{2}$ and $\sigma_{B(h)}^{2}$ in Eq.(2), respectively.

$$
\left\{\begin{array}{l}
\left\{r_{1}^{*}, \ldots, r_{K-1}^{*}\right\}=\underset{0 \leq r_{1}<\cdots<r_{K-1} \leq L-1}{\arg \max }\left\{\sigma_{B(f)}^{2}\left(r_{1}, \ldots, r_{K-1}\right)\right\} \\
\left\{s_{1}^{*}, \ldots, s_{K-1}^{*}\right\}=\underset{0 \leq s_{1}<\cdots<s_{K-1} \leq L-1}{\arg \max }\left\{\sigma_{B(g)}^{2}\left(s_{1}, \ldots, s_{K-1}\right)\right\} \\
\left\{t_{1}^{*}, \ldots, t_{K-1}^{*}\right\}=\underset{0 \leq t_{1}<\cdots<t_{K-1} \leq L-1}{\arg \max }\left\{\sigma_{B(h)}^{2}\left(t_{1}, \ldots, t_{K-1}\right)\right\}
\end{array}\right.
$$

Finally, we use the threshold set $\left\{\frac{r_{1}^{*}+s_{1}{ }^{*}+t_{1}^{*}}{3}, \ldots, \frac{r_{K-1}{ }^{*}+s_{K-1}{ }^{*}+t_{K-1}{ }^{*}}{3}\right\}$ to segment the original image $I$.

The time complexity of the efficient 3D Otsu bi-level thresholding is $O(L)$. For multilevel thresholding, the time complexity will be $O\left(L^{K-1}\right)$, where $(K-1)$ denotes the number of thresholds.

\subsection{Segmentation map fusion}

Assume the decomposition scale is $l$, there will be $l$ segmentation map, namely $\left\{R_{0}, R_{1}, \ldots, R_{l}\right\}$. Generally, most of the pixels in the source image are given the same labels in these segmentation maps, here let $P=\left\{\mathbf{p} \mid \mathbf{p}=\underset{\mathbf{p}}{\arg }\left\{R_{0}(\mathbf{p})=R_{1}(\mathbf{p})=\ldots=R_{l}(\mathbf{p})\right\}\right\}$ denote such pixels' locations, the locations of rest of pixels whose labels are different in different segmentation maps are kept in $Q$. Fig.4 shows a bi-level image segmentation case where there are three segmentation maps, and the figure illustrates how to determine the sets $P$ and $Q$. 
(a) $R_{0}$

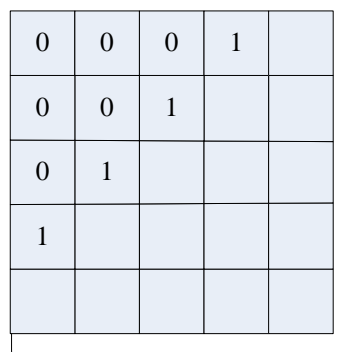

(b) $R_{1}$

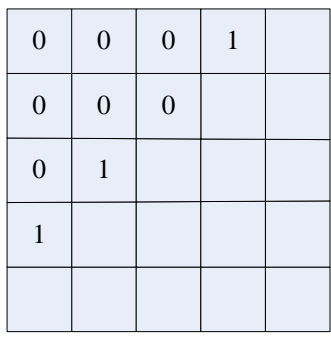

(c) $R_{2}$

\begin{tabular}{|c|c|c|c|c|}
\hline 0 & 0 & 0 & 0 & \\
\hline 0 & 0 & 1 & & \\
\hline 0 & 1 & & & \\
\hline 1 & & & & \\
\hline & & & & \\
\hline
\end{tabular}

$P=\{(1,1),(1,2),(1,3)$,

$(2,1),(2,2),(3,1)$

$(3,2),(4,1), \ldots\}$;

$Q=\{(1,4),(2,3)\}$

(e)

Fig.4 Pixel classification based on whether their labels on different segmentation maps are the same: (a), (b) and (c) are three segmentation maps; (d) pixel classification maps: a red block with "+" means the corresponding pixel that is segmented into the same region in (a), (b) and (c), and a green block with "-" corresponds a pixel that is segmented into different regions; (e) the sets $P$ and $Q$.

For the pixel $\mathbf{p} \in P$, its final segmentation label is

$$
R(\mathbf{p})=R_{0}(\mathbf{p})
$$

Based on $R(P)$, the means of the segments can be calculated which are denoted by $\left\{m_{1}, \ldots, m_{K}\right\}$.

For the pixel $\mathbf{q} \in Q$, its final segmentation label is

$$
R(\mathbf{q})=R_{\hat{i}}(\mathbf{q}) .
$$

Here,

$$
\hat{i}=\underset{i}{\arg \min }\left\{\left|f(\mathbf{q})-m_{R_{i}}\right|\right\} \quad i=0, \ldots s c a
$$

where $f(\mathbf{q})$ refers to the gray level of the pixel $\mathbf{q}$ in the original image.

From Eq.(7) and (8), it can be noted that the final segmentation labels of the pixels in $Q$ are determined by their distances from the mean of each region: they are labeled by the region whose mean is closest to the pixels' gray level.

\section{Experimental results and analysis}

\subsection{Experimental settings}

The performance of the proposed algorithm is demonstrated on medical brain images which are freely available on the website (http://www.med.harvard.edu/aanlib/home.html) of Harvard Medical 
School. Here, we choose ten MR-T2 brain slices as shown in Fig.5. In the proposed algorithm, the number of thresholds $(K-1)$ is ranging from 1 to $5 .(K-1)=1$ is set for single thresholding segmentation (shown in subsection 4.2), while $(K-1)=2,3,4,5$ are selected for the multilevel thresholding segmentation (shown in subsection 4.3).

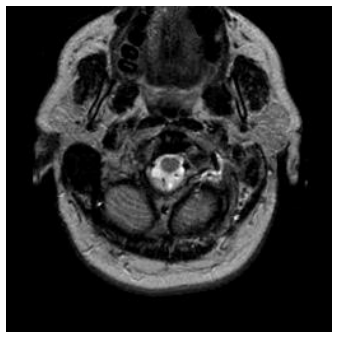

(a) slice \#22

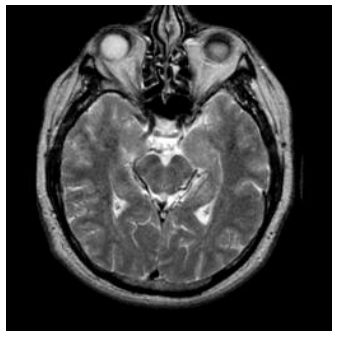

(e) slice \#62

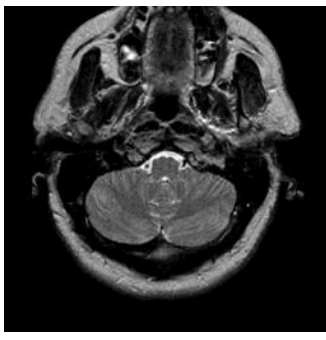

(b) slice \#32

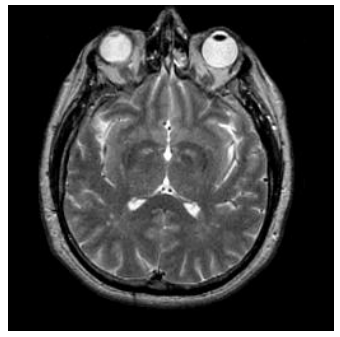

(f) slice \#72

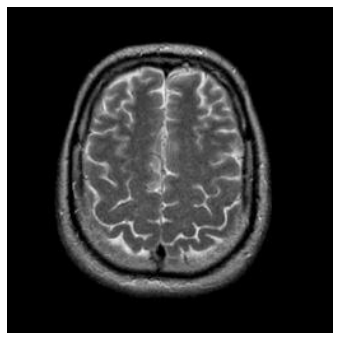

(i) slice \#102

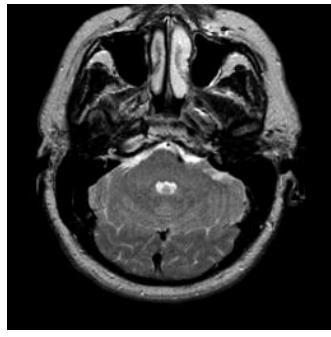

(c) slice \#42

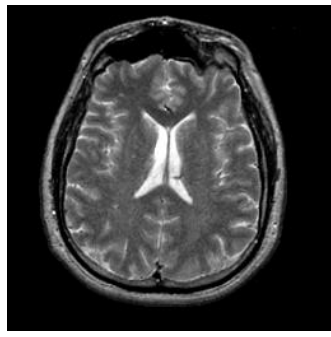

(g) slice \#82

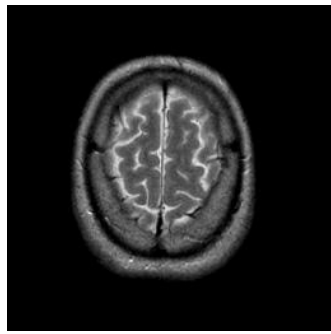

(j) slice \#112

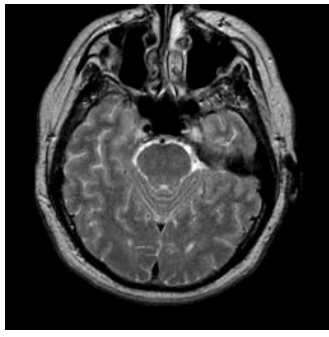

(d) slice \#52

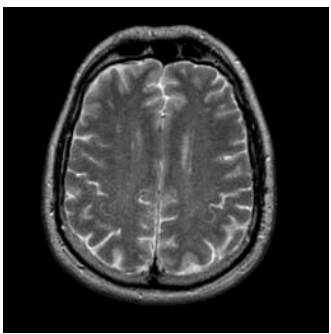

(h) slice \#92

Fig. 5 MR-T2 brain slices.

In addition, uniformity measure $[15,17]$ is employed to evaluate segmentation results precisely in this paper. For a segmented image, the uniformity measure is defined as,

$$
u=1-2 *(K-1) * \frac{\sum_{j=1}^{K} \sum_{i \in R_{j}}\left(f_{i}-\mu_{j}\right)^{2}}{N u m *\left(f_{\max }-f_{\min }\right)}
$$

where $K$ denotes the number of classes segmented by the thresholding; Num represents the total number of pixels; $f_{\max }$ and $f_{\min }$ denote the maximum gray level and the minimum gray level of the pixels in the image, respectively; $R_{j}$ indicates $j_{\text {th }}$ segmented region; $f_{i}$ denotes the gray level of pixel $i$, and $\mu_{j}$ refers to the mean gray level of pixels in region $R_{j}$. The value of the uniformity measure $u$ is varying from 0 to 1 . A higher value of $u$ means better uniformity in the segmented image and better performance of the thresholding, and vice versa. 


\subsection{Scale size selection}

The decomposition scale is an important parameter affecting the algorithm's performance. For the selection of scale size, we test the brain slices in three cases: scale $=0,1,2,3$. The evaluation of segmentation results on different scales is illustrated in Table 1. The table encompasses the uniformity measure values and objective values for each slice with different scales and different number of thresholds.

Table 1 Evaluation of segmentation results on different scales

\begin{tabular}{|c|c|c|c|c|c|c|c|c|c|}
\hline \multirow{3}{*}{$\begin{array}{l}\text { Testing } \\
\text { images }\end{array}$} & \multirow{3}{*}{$\begin{array}{c}\text { Number of } \\
\text { thresholds } \\
(K-1) \\
1\end{array}$} & \multicolumn{8}{|c|}{ Uniformity measure $(u) \&$ objective value } \\
\hline & & \multicolumn{2}{|c|}{ scale $=0$} & \multicolumn{2}{|c|}{ scale $=1$} & \multicolumn{2}{|c|}{ scale $=2$} & \multicolumn{2}{|c|}{ scale $=3$} \\
\hline & & 0.9845 & 501.8862 & 0.9849 & 1987.60 & 0.9849 & 1987.60 & 0.9806 & 1848.40 \\
\hline \multirow{4}{*}{$\begin{array}{l}\text { Slice } \\
\# 022\end{array}$} & 2 & 0.9848 & 909.5362 & 0.9863 & 2256.80 & 0.9863 & 2257.50 & 0.9829 & 2202.20 \\
\hline & 3 & 0.9316 & 1039.739 & 0.9802 & 2265.90 & 0.9810 & 2273.70 & 0.9786 & 2248.60 \\
\hline & 4 & 0.9018 & 726.4376 & 0.9820 & 2333.80 & 0.9850 & 2357.90 & 0.9807 & 2323.50 \\
\hline & 5 & 0.8615 & 721.9398 & 0.9832 & 2371.00 & 0.9875 & 2398.70 & 0.9819 & 2362.50 \\
\hline \multirow{5}{*}{$\begin{array}{l}\text { Slice } \\
\# 032\end{array}$} & 1 & 0.9839 & 693.1817 & 0.9840 & 2312.50 & 0.9840 & 2312.80 & 0.9822 & 2254.90 \\
\hline & 2 & 0.9837 & 1240.56 & 0.9851 & 2590.90 & 0.9851 & 2591.60 & 0.9816 & 2533.60 \\
\hline & 3 & 0.9390 & 1311.85 & 0.9869 & 2691.80 & 0.9874 & 2696.30 & 0.9791 & 2607.00 \\
\hline & 4 & 0.9289 & 1730.553 & 0.9857 & 2716.90 & 0.9858 & 2717.80 & 0.9786 & 2659.10 \\
\hline & 5 & 0.8520 & 869.8737 & 0.9833 & 2724.50 & 0.9839 & 2728.70 & 0.9811 & 2710.60 \\
\hline \multirow{5}{*}{$\begin{array}{l}\text { Slice } \\
\# 042\end{array}$} & 1 & 0.9797 & 880.2393 & 0.9798 & 2643.90 & 0.9798 & 2644.30 & 0.9780 & 2588.00 \\
\hline & 2 & 0.9812 & 1485.832 & 0.9831 & 3027.30 & 0.9831 & 3027.70 & 0.9778 & 2940.50 \\
\hline & 3 & 0.9373 & 1556.091 & 0.9838 & 3126.00 & 0.9838 & 3126.40 & 0.9789 & 3073.00 \\
\hline & 4 & 0.8929 & 1852.278 & 0.9849 & 3179.00 & 0.9850 & 3180.10 & 0.9793 & 3133.80 \\
\hline & 5 & 0.8939 & 2165.41 & 0.9861 & 3211.10 & 0.9865 & 3214.30 & 0.9795 & 3168.20 \\
\hline \multirow{5}{*}{$\begin{array}{l}\text { Slice } \\
\# 052\end{array}$} & 1 & 0.9827 & 1044.688 & 0.9827 & 2521.10 & 0.9827 & 2523.30 & 0.9820 & 2500.40 \\
\hline & 2 & 0.9832 & 1548.114 & 0.9850 & 2840.70 & 0.9850 & 2841.70 & 0.9810 & 2776.40 \\
\hline & 3 & 0.9450 & 1657.768 & 0.9842 & 2914.20 & 0.9855 & 2928.30 & 0.9807 & 2875.50 \\
\hline & 4 & 0.8946 & 1194.056 & 0.9846 & 2960.10 & 0.9857 & 2969.00 & 0.9810 & 2930.50 \\
\hline & 5 & 0.8822 & 1243.17 & 0.9857 & 2992.20 & 0.9865 & 2997.50 & 0.9785 & 2945.40 \\
\hline \multirow{5}{*}{$\begin{array}{l}\text { Slice } \\
\# 062\end{array}$} & 1 & 0.9764 & 1289.566 & 0.9764 & 2888.70 & 0.9765 & 2890.50 & 0.9754 & 2854.10 \\
\hline & 2 & 0.9798 & 1789.3 & 0.9814 & 3351.40 & 0.9814 & 3351.60 & 0.9752 & 3251.70 \\
\hline & 3 & 0.9465 & 2089.829 & 0.9826 & 3465.50 & 0.9826 & 3465.70 & 0.9759 & 3393.30 \\
\hline & 4 & 0.9007 & 1566.853 & 0.9829 & 3515.30 & 0.9831 & 3517.10 & 0.9769 & 3466.60 \\
\hline & 5 & 0.8795 & 2385.986 & 0.9847 & 3555.30 & 0.9851 & 3557.40 & 0.9719 & 3472.00 \\
\hline
\end{tabular}


Table 1 (Continued)

\begin{tabular}{|c|c|c|c|c|c|c|c|c|c|}
\hline \multirow{2}{*}{$\begin{array}{l}\text { Testing } \\
\text { images }\end{array}$} & \multirow{2}{*}{$\begin{array}{c}\text { Number of } \\
\text { thresholds } \\
(K-1)\end{array}$} & \multicolumn{8}{|c|}{ Uniformity measure $(u) \&$ objective value } \\
\hline & & \multicolumn{2}{|c|}{ Scale $=0$} & \multicolumn{2}{|c|}{ Scale $=1$} & \multicolumn{2}{|c|}{ Scale $=2$} & \multicolumn{2}{|c|}{ Scale $=3$} \\
\hline \multirow{5}{*}{$\begin{array}{l}\text { Slice } \\
\# 072\end{array}$} & 1 & 0.9749 & 1231.626 & 0.9747 & 2688.80 & 0.9748 & 2693.80 & 0.9741 & 2670.00 \\
\hline & 2 & 0.9785 & 1813.407 & 0.9800 & 3186.00 & 0.9800 & 3187.10 & 0.9742 & 3093.30 \\
\hline & 3 & 0.9498 & 2275.429 & 0.9822 & 3319.50 & 0.9824 & 3321.20 & 0.9775 & 3268.10 \\
\hline & 4 & 0.9278 & 2399.759 & 0.9843 & 3384.20 & 0.9844 & 3385.20 & 0.9765 & 3320.90 \\
\hline & 5 & 0.8698 & 1645.186 & 0.9821 & 3395.70 & 0.9848 & 3413.30 & 0.9761 & 3356.20 \\
\hline \multirow{5}{*}{$\begin{array}{l}\text { Slice } \\
\# 082\end{array}$} & 1 & 0.9794 & 1102.484 & 0.9795 & 2547.40 & 0.9795 & 2548.50 & 0.9426 & 2527.50 \\
\hline & 2 & 0.9808 & 1779.085 & 0.9821 & 2924.80 & 0.9821 & 2925.00 & 0.9753 & 2815.50 \\
\hline & 3 & 0.9581 & 2273.664 & 0.9843 & 3046.10 & 0.9843 & 3046.80 & 0.9820 & 3021.80 \\
\hline & 4 & 0.9169 & 2099.552 & 0.9832 & 3080.20 & 0.9856 & 3099.70 & 0.9777 & 3035.20 \\
\hline & 5 & 0.8727 & 1553.743 & 0.9836 & 3109.90 & 0.9855 & 3122.40 & 0.9791 & 3080.30 \\
\hline \multirow{5}{*}{$\begin{array}{l}\text { Slice } \\
\# 092\end{array}$} & 1 & 0.9858 & 976.2127 & 0.9860 & 2367.60 & 0.9860 & 2367.60 & 0.9842 & 2308.60 \\
\hline & 2 & 0.9875 & 1708.327 & 0.9890 & 2642.90 & 0.9890 & 2642.90 & 0.9805 & 2504.30 \\
\hline & 3 & 0.9741 & 1739.281 & 0.9893 & 2705.10 & 0.9893 & 2705.40 & 0.9826 & 2632.40 \\
\hline & 4 & 0.9309 & 1272.38 & 0.9890 & 2732.20 & 0.9896 & 2736.70 & 0.9807 & 2664.30 \\
\hline & 5 & 0.9063 & 1102.353 & 0.9886 & 2747.30 & 0.9901 & 2756.80 & 0.9819 & 2703.50 \\
\hline \multirow{5}{*}{$\begin{array}{l}\text { Slice } \\
\# 102\end{array}$} & 1 & 0.9856 & 792.2598 & 0.9858 & 2297.10 & 0.9858 & 2297.20 & 0.9841 & 2240.50 \\
\hline & 2 & 0.9865 & 1639.729 & 0.9882 & 2560.10 & 0.9882 & 2560.20 & 0.9819 & 2459.70 \\
\hline & 3 & 0.9734 & 1717.994 & 0.9891 & 2631.40 & 0.9892 & 2632.70 & 0.9824 & 2560.40 \\
\hline & 4 & 0.9376 & 1223.514 & 0.9866 & 2640.80 & 0.9884 & 2655.10 & 0.9792 & 2581.70 \\
\hline & 5 & 0.9193 & 1203.539 & 0.9877 & 2668.90 & 0.9884 & 2673.20 & 0.9781 & 2608.00 \\
\hline \multirow{5}{*}{$\begin{array}{l}\text { Slice } \\
\# 112\end{array}$} & 1 & 0.9883 & 413.702 & 0.9886 & 1819.40 & 0.9886 & 1819.70 & 0.9867 & 1759.50 \\
\hline & 2 & 0.9882 & 1264.765 & 0.9889 & 2002.60 & 0.9891 & 2005.30 & 0.9871 & 1973.50 \\
\hline & 3 & 0.9762 & 1291.91 & 0.9909 & 2081.30 & 0.9910 & 2081.60 & 0.9842 & 2011.10 \\
\hline & 4 & 0.9599 & 1482.094 & 0.9918 & 2111.60 & 0.9919 & 2112.40 & 0.9846 & 2055.60 \\
\hline & 5 & 0.9696 & 1407.697 & 0.9911 & 2120.10 & 0.9919 & 2124.80 & 0.9838 & 2074.40 \\
\hline
\end{tabular}

Note that "scale $=0$ " means single-scale 3D Otsu algorithm, namely the algorithm contains no multi-scale image representation. From Table 1, it can be observed that the objective values of all the brain slices in the cases of "scale $=1$ ", "scale $=2$ " and "scale $=3$ " are significantly higher than those in the case of "scale $=0$ ", as well as the uniformity measure values. The experimental results demonstrate that multi-scale image representation is conducive to get more precise segmentations.

Besides, among cases of "scale=1", "scale=2" and "scale=3", the former two perform better. The evaluation values of "scale $=1$ " and "scale $=2$ " are close, but generally the former case is superior to the later one. Taking slice $\# 022$ as an example, the uniformity measure values of "scale $=1$ " and "scale $=2$ " are the same when $(\mathrm{K}-1)=1$ and $(\mathrm{K}-1)=2$. For $(\mathrm{K}-1)=3,4,5$, the evaluation values of "scale $=2$ " are slightly higher than those of "scale=1"; the differences of uniformity measure values are $0.0008,0.003$ and 0.0043 , respectively; and the differences of the objective values are 7.8, 24.1 and 27.7, respectively. It indicates that the segmentation results of "scale=2" are increasingly better than those of "scale=1" with the number of thresholds (K-1) increasing, that is the final segmentation result obtained by fusing the initial segmentation result $\mathrm{R} 0$ and once-FLLF-based segmentation result $\mathrm{R} 1$ is the best in current. Therefore, we choose the case of "scale=2" in the following experiments. 


\subsection{Single thresholding segmentation}

\subsubsection{Single thresholding segmentation}

To verify the performance of the proposed multi-scale efficient 3D Otsu thresholding algorithm (MS3D Otsu), the traditional one-dimensional Otsu thresholding (1D Otsu) and the three-dimensional reconstruction and dimension reduction based Otsu thresholding (RR3D Otsu) [39] are utilized for comparison in terms of single thresholding segmentation.

Slice

\#022
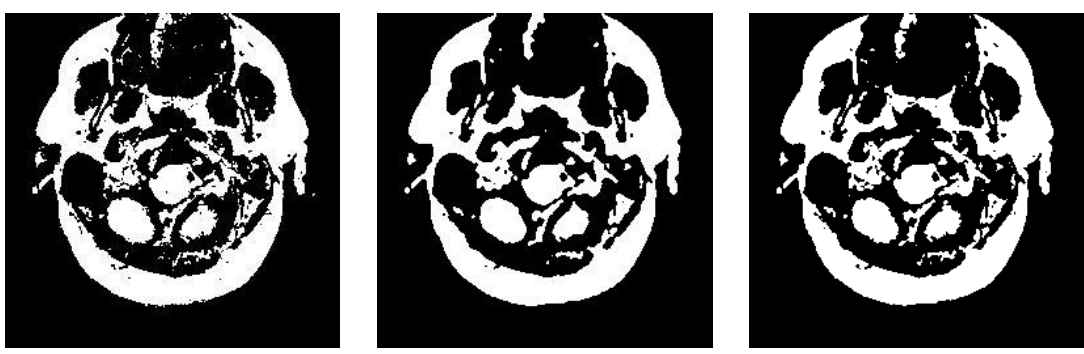

Slice
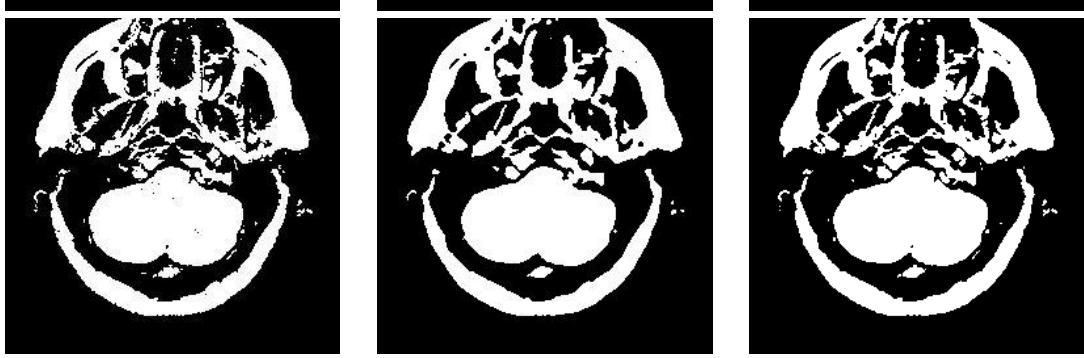

\#032
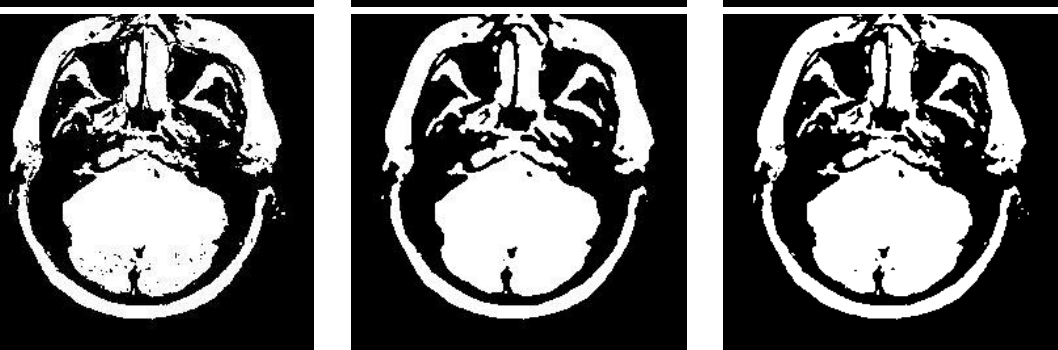

Slice

\#042
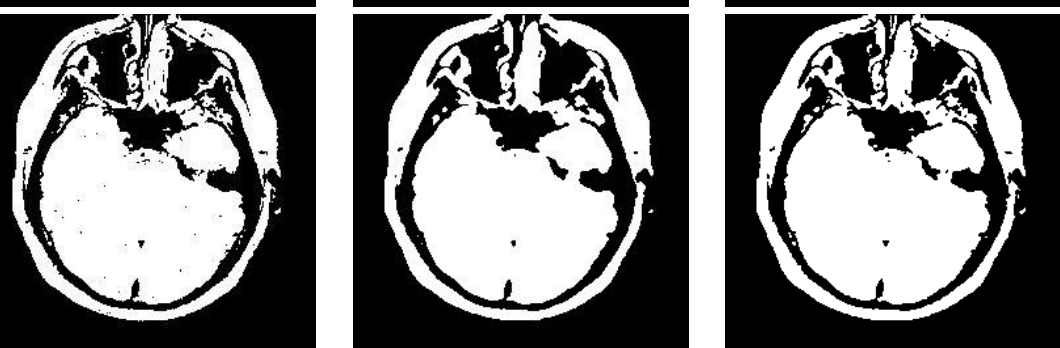

Slice

$\# 052$

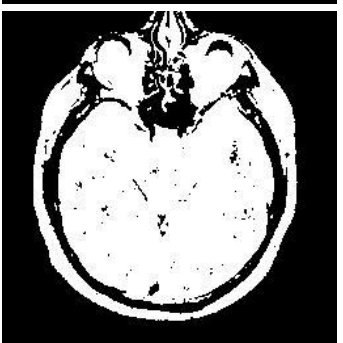

(a) 1D Otsu

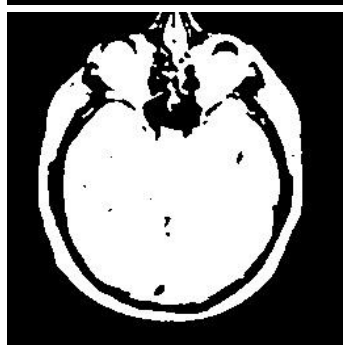

(b) RR3D Otsu

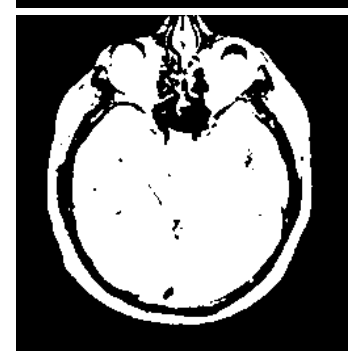

(c) MS3D Otsu

Fig. 6 Segmentation results of brain slices \#022 \#112 (from top to bottom) by different single thresholding algorithms: (a) 1D Otsu, (b) RR3D Otsu, (c) MS3D Otsu. 

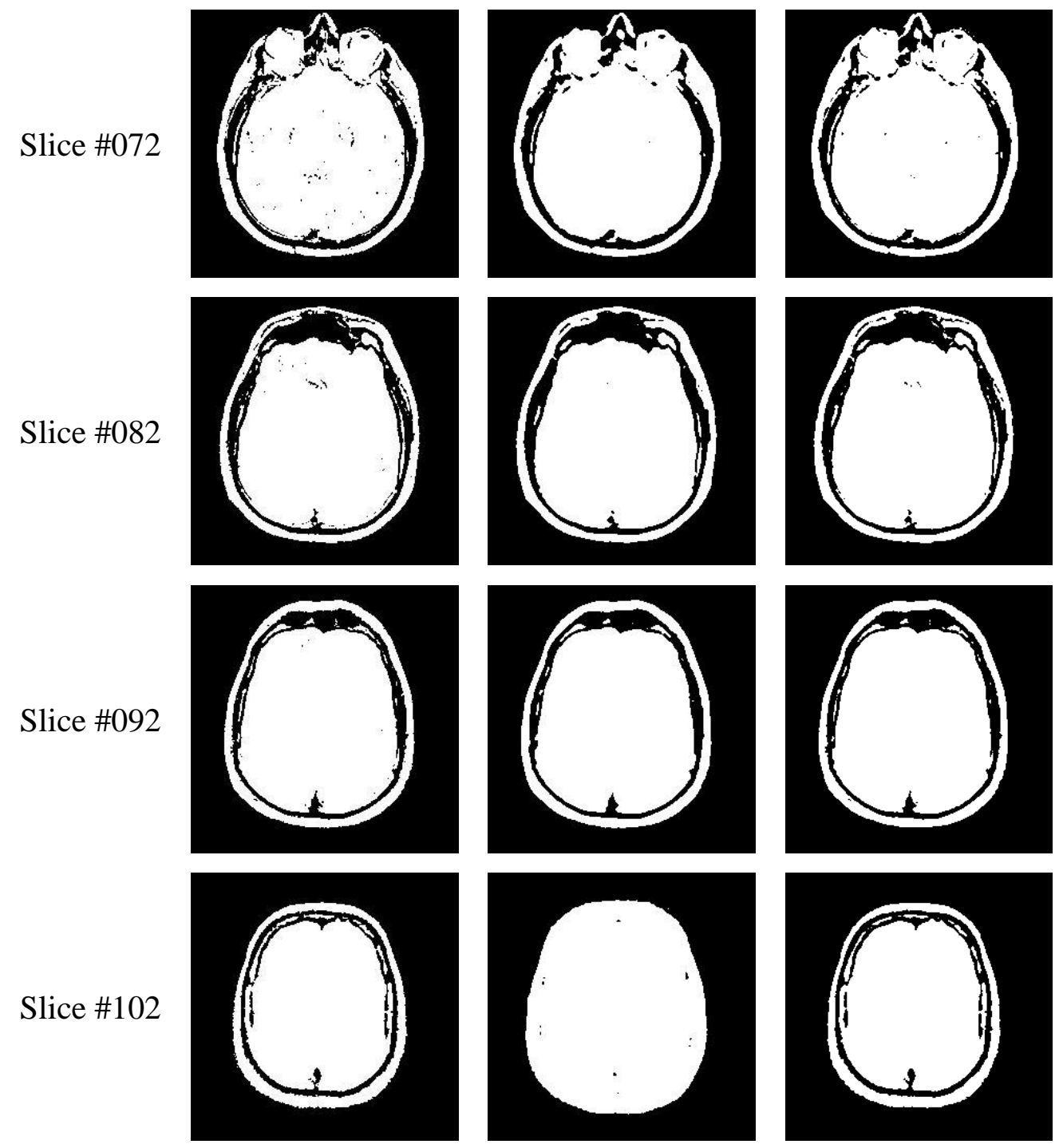

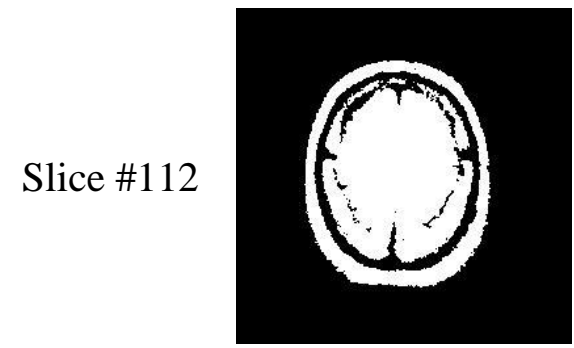

(a) 1D Otsu

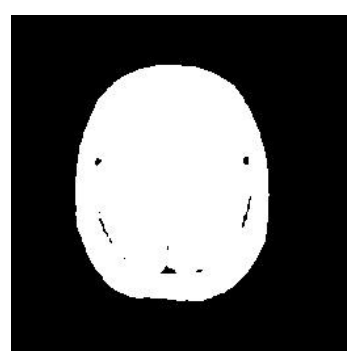

(b) RR3D Otsu

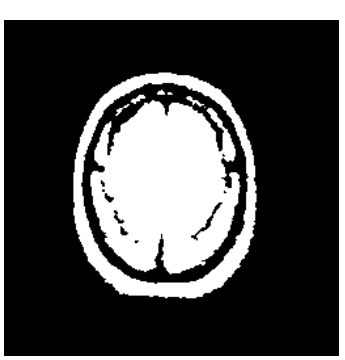

(c) MS3D Otsu

Fig. 6 (Continued)

The segmentation results obtained by the three different thresholding methods are displayed in Fig.6. In the figure, the segmented images of brain slices \#022 \#112 are shown from top to bottom. The three groups Fig.6(a) (c) are the results gained by 1D Otsu, RR3D Otsu and MS3D Otsu, respectively. In Fig.6(a), it can be noted that many fragmented small regions (namely misclassified pixels or noise points) are existed in the segmentation results, such as slice \#022 and slices \#052 \#072. In contrast, there is hardly any noise in group Fig.6(b), which exactly verifies the denoising performance of 3D Otsu. However, some tissue regions in the brain slices are failed to be segmented, especially for slice \#102 and slice \#112. This is because the manipulation of 
dimensionality reduction in the RR3D Otsu method will inevitably cause information loss. In Fig.6(c), it can be seen that the tissue regions of brain slices are clearly segmented with relatively less noise. Visually, the segmentation results obtained by our proposed MS3D Otsu method are better than others.

To analyze the segmentation results quantitively, we give the comparison of uniformity measure for the three methods as shown in Table 2. The best results have been marked in bold. From the table, it can be observed that, except for the slice \#092, MS3D Otsu thresholding gives the highest value to each of the rest nine brain slices. For slice \#092, the segmented image by traditional 1D Otsu thresholding gains the highest uniformity measure value, but it is only 0.0001 higher than the result given by MS3D Otsu thresholding. Therefore, the proposed method in this paper is superior to the other two methods in the aspect of single thresholding segmentation.

Table 2 Comparison of the uniformity measure for different single thresholding algorithms

\begin{tabular}{cccccccccccc}
\hline Methods & \multicolumn{8}{c}{ Testing images (slice \#022 slice \#112) } \\
\hline 1D Otsu & 0.9849 & 0.9840 & 0.9798 & 0.9827 & 0.9765 & 0.9748 & 0.9795 & $\mathbf{0 . 9 8 6 1}$ & 0.9858 & 0.9886 \\
RR3D & 0.9841 & 0.9831 & 0.9789 & 0.9820 & 0.9756 & 0.9741 & 0.9790 & 0.9858 & 0.9727 & 0.9789 \\
Otsu & & & & & & & & & & \\
MS3D & $\mathbf{0 . 9 8 5 2}$ & $\mathbf{0 . 9 8 4 3}$ & $\mathbf{0 . 9 8 0 1}$ & $\mathbf{0 . 9 8 3 1}$ & $\mathbf{0 . 9 7 6 8}$ & $\mathbf{0 . 9 7 5 2}$ & $\mathbf{0 . 9 7 9 6}$ & 0.9860 & $\mathbf{0 . 9 8 5 9}$ & $\mathbf{0 . 9 8 8 8}$ \\
Otsu & & & & & & & & & & \\
\hline
\end{tabular}

\subsubsection{Single thresholding segmentation for noise images}

In real applications, the medical images may contain some noises to some extent. Hence, it is necessary to examine whether the proposed segmentation algorithm can process noised images. Fig.7 displays four noised images which are generated by adding Gaussian noises $N(0,0.01)$ to original images. The four noised images are used for test the proposed algorithm.

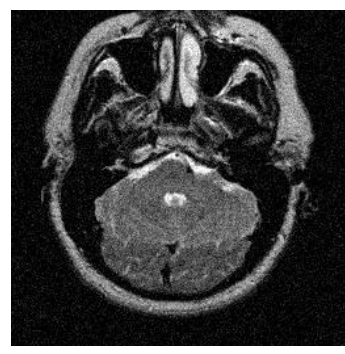

Slice \#042

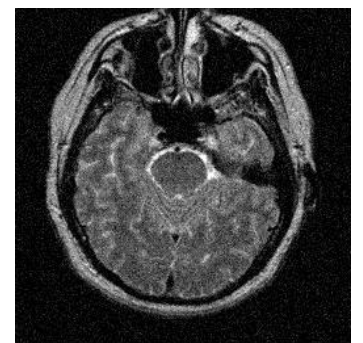

Slice \#052

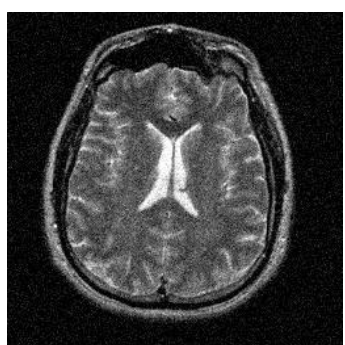

Slice \#082

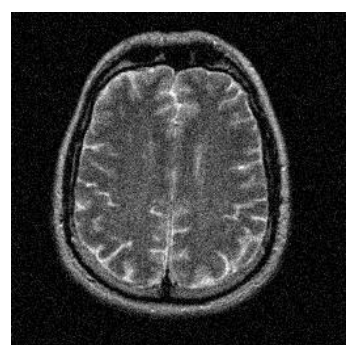

Slice \#092

Fig.7 Four noised medical images

The segmentation results by 1D Otsu, RR3D Otsu and the proposed MS3D Otsu are shown in Fig.8. It can be observed that the segmentation results by 1D Otsu are heavily affected by noise, especially in the soft tissue regions. RR3D Otsu performs better than 1D Otsu, it can reduce the influence of noises because it takes spatial information into account. By contrast, the proposed algorithm can effectively avoid noises. The uniformity measure for different single thresholding algorithms listed 
in Table 3 also shows that the proposed algorithm performs the best among the three comparative algorithms consistently.

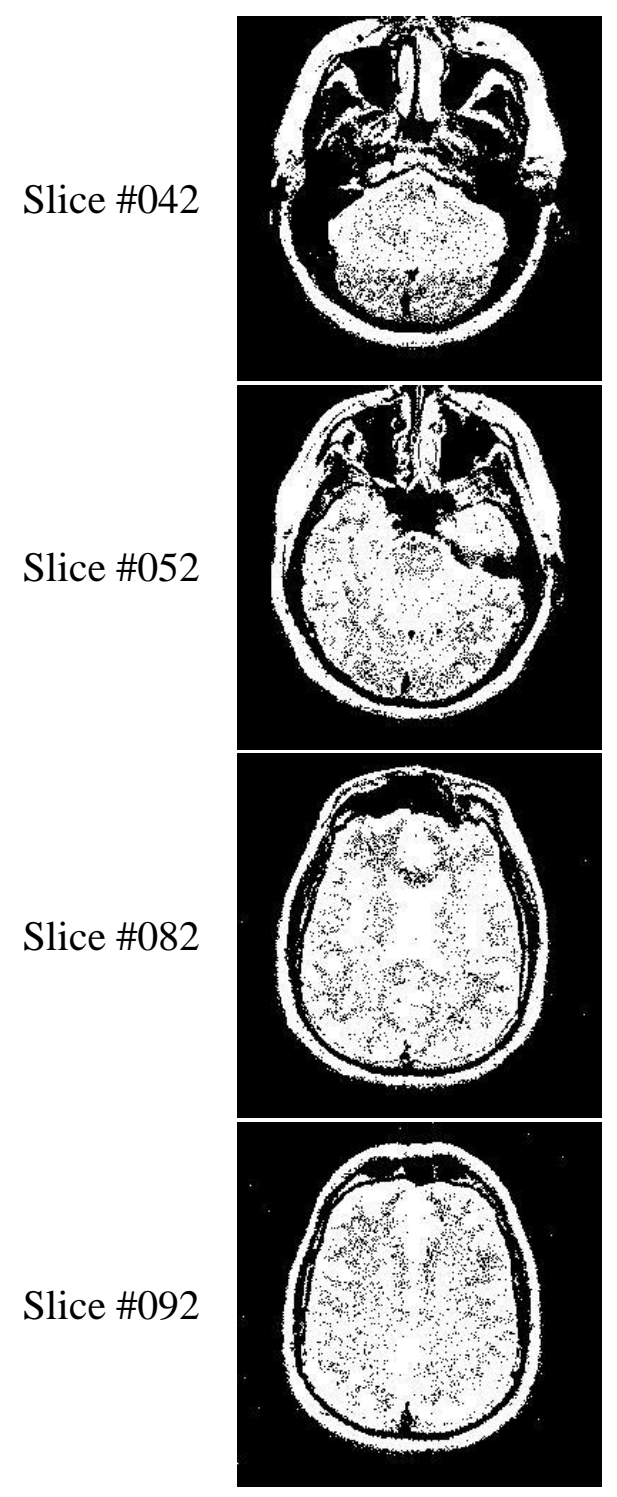

(a) 1 D Otsu
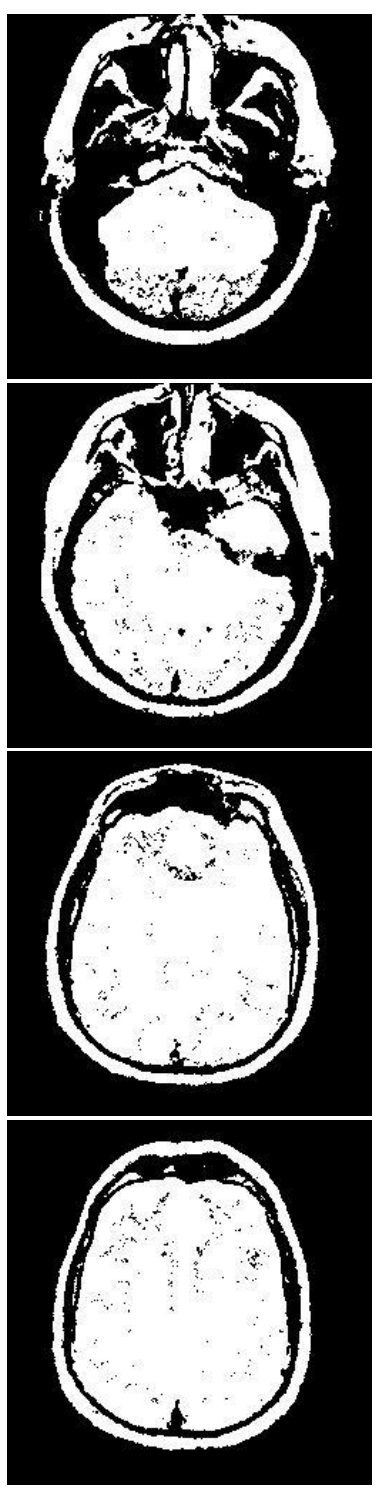

(b) RR3D Otsu
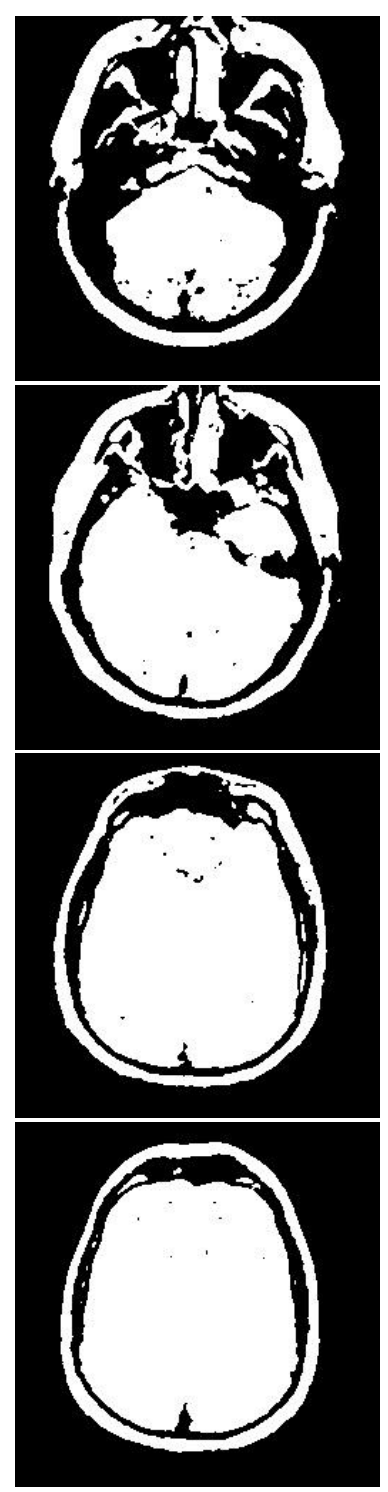

(c) MS3D Otsu

Fig.8 Segmentation results of noised medical images

Table 3 Comparison of the uniformity measure for different single thresholding algorithms

\begin{tabular}{ccccc}
\hline \multirow{2}{*}{ Methods } & \multicolumn{4}{c}{ Testing images } \\
\cline { 2 - 5 } & slice \#042 & slice \#052 & slice \#082 & slice \#092 \\
\hline (a) 1D Otsu & 0.9753 & 0.9777 & 0.9740 & 0.9809 \\
(b) RR 3D Otsu & 0.9783 & 0.9816 & 0.9786 & 0.9855 \\
(c) MS3D Otsu & $\mathbf{0 . 9 7 8 7}$ & $\mathbf{0 . 9 8 1 8}$ & $\mathbf{0 . 9 7 8 5}$ & $\mathbf{0 . 9 8 5 2}$ \\
\hline
\end{tabular}

\subsection{Multilevel thresholding segmentation}

In the case of $(K-1)=2,3,4,5$, the proposed method is compared with four popular multilevel thresholding approaches, including particle swarm optimization (PSO) [40] / bacterial foraging (BF) 
[18]/adaptive bacterial foraging (ABF) [17] based method, and multilevel thresholding using real coded genetic algorithm (RGA) [16].

The segmentation results of brain slices \#022 \#112 obtained by our proposed algorithm (MS3D Otsu) with various numbers of thresholds are shown from top to bottom in Fig. 9. Subjective evaluation indicates that the segmented images provide clear gray gradation and nice visual effect. Intensity consistency in each segmented region is maintained well as the number of thresholds $(K-1)$ increases. Objective evaluation of the segmentation results obtained by MS3D Otsu and other four multilevel thresholding methods (PSO, BF, ABF, RGA) are depicted in Table 4 and Table 5, where the best values have been marked in bold.
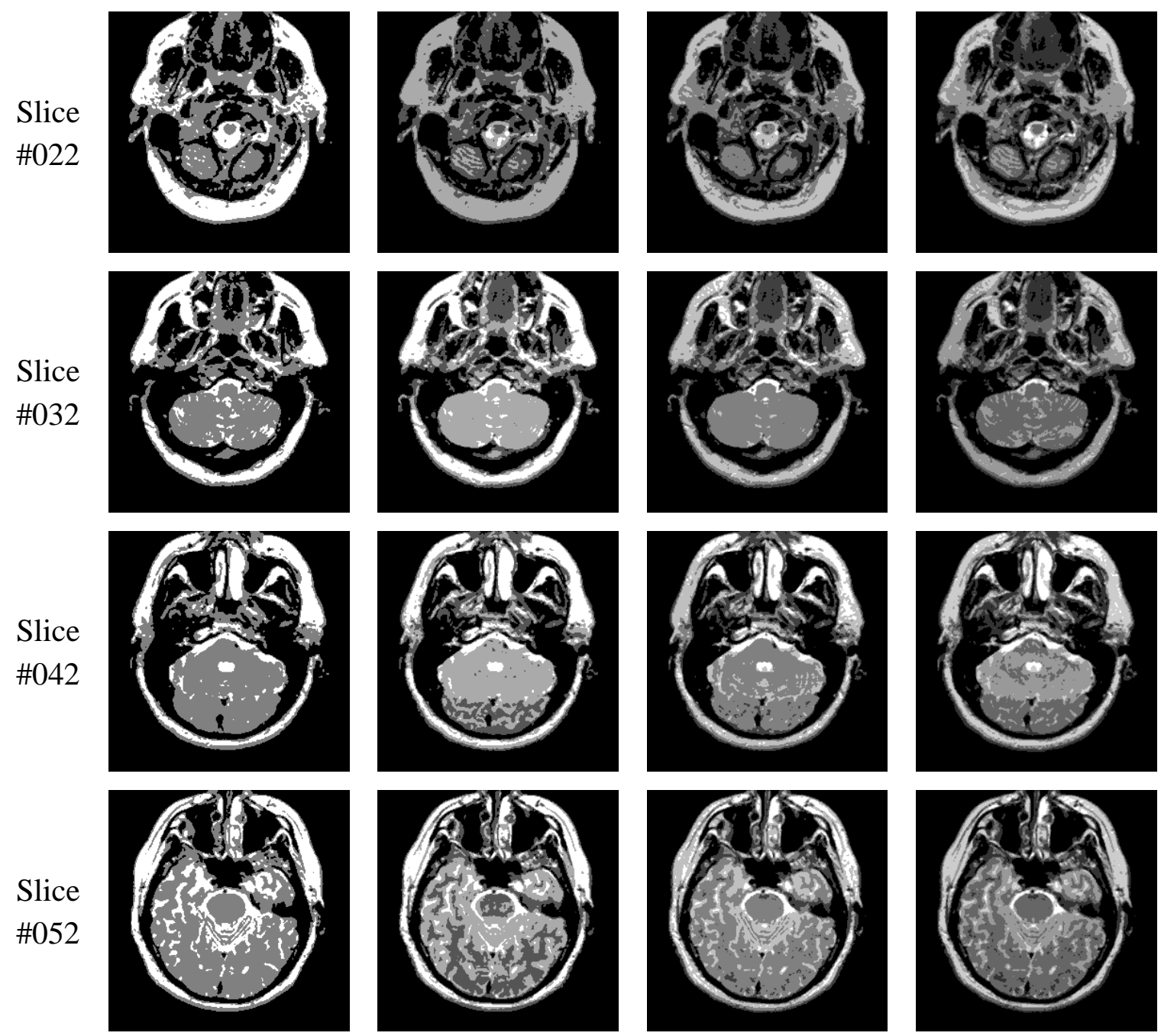

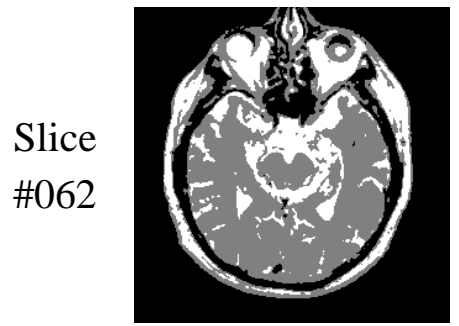

(a) $(K-1)=2$

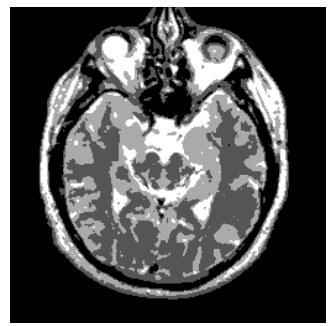

(b) $(K-1)=3$

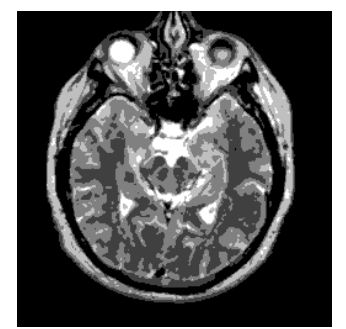

(c) $(K-1)=4$

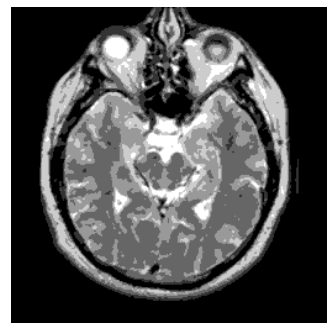

(d) $(K-1)=5$

Fig. 9 Multilevel thresholding segmentation results of brain slices \#022 \#112 (from top to bottom) by the proposed algorithm 

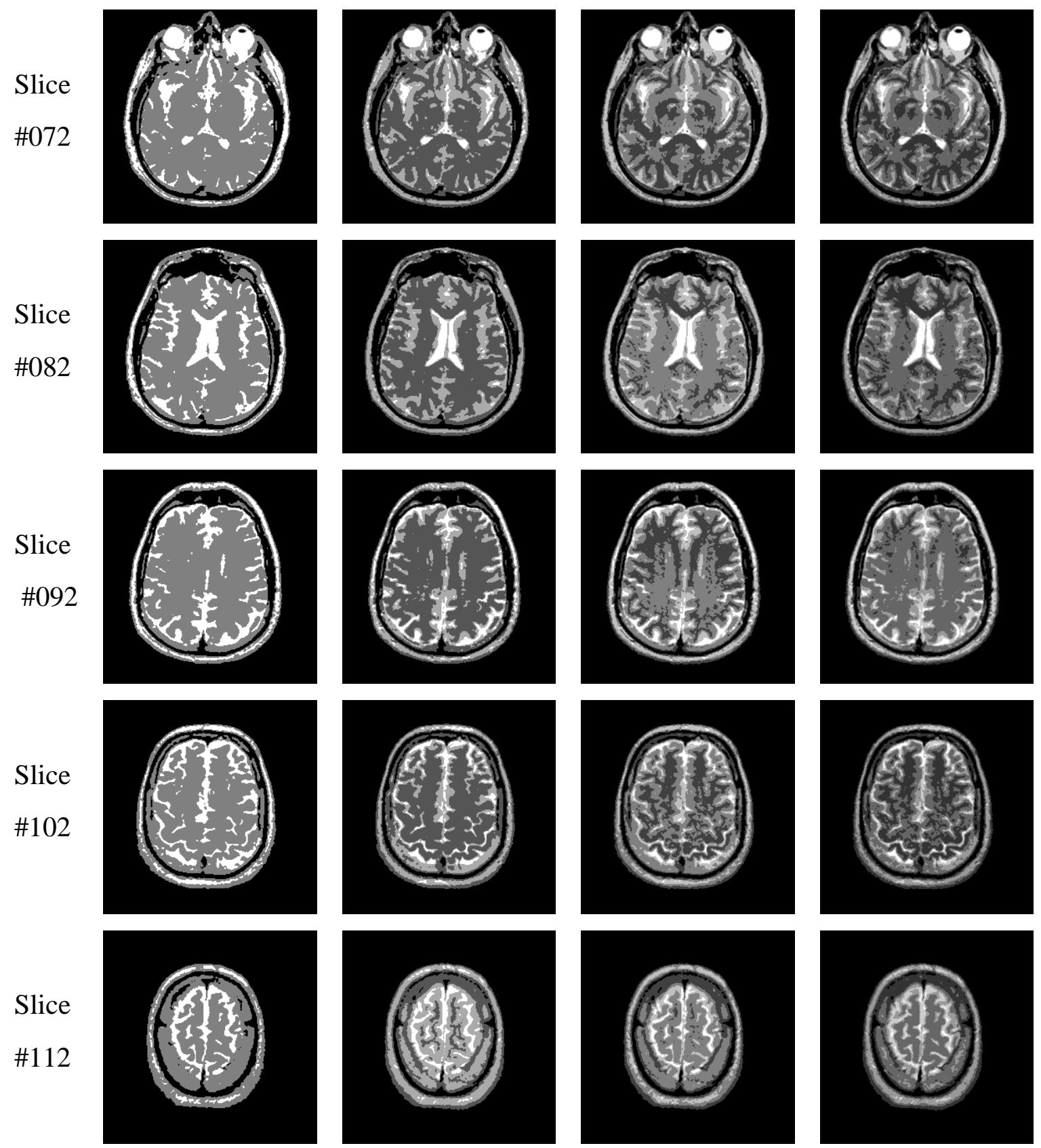

(a) $(K-1)=2$

(b) $(K-1)=3$

(c) $(K-1)=4$

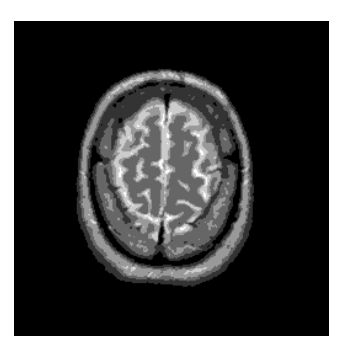

Fig. 9 (Continued)

Table 4 reveals that MS3D Otsu has obtained the maximum between-class variance for almost all the brain slices and different numbers of threshold $(K-1)$. Especially for the slices \#062 \#102, the objective values obtained by MS3D Otsu are significantly higher than those obtained by PSO, BF, $\mathrm{ABF}$ and RGA. Furthermore, Table 5 explores the comparison of uniformity measure values obtained with the five algorithms, which indicates that MS3D Otsu has realized higher values than others for almost all the images with every $(K-1)$. Similarly, for slices \#062 \#102 $((K-1)=2,3,4)$, segmentation results obtained by MS3D Otsu are overwhelmingly better than those obtained by other four algorithms. In other words, it can be evidently noted that the uniformity measure results also confirm the results of objective values. Just for the segmentation results: slice \#032 with $(K-1)=5$, slice \#042 with $(K-1)=4,5$, and slice \#052 with $(K-1)=5$, their uniformity measure values obtained 
by RGA are higher than those obtained by MS3D Otsu. However, MS3D Otsu has obtained acceptable evaluation results, and the differences for these slices between RGA and MS3D Otsu are 0.0004, 0.0015, 0.0012, 0.0003, respectively. Generally, RGA, ABF, BF and PSO follow the MS3D Otsu's success.

Table 4 Comparison of objective values for different segmentation methods

\begin{tabular}{|c|c|c|c|c|c|c|}
\hline \multirow{2}{*}{$\begin{array}{l}\text { Testing } \\
\text { images }\end{array}$} & \multirow{2}{*}{$\begin{array}{c}\text { Number of } \\
\text { thresholds }(K-1)\end{array}$} & \multicolumn{5}{|c|}{ Objective values } \\
\hline & & PSO & $\mathrm{BF}$ & $\mathrm{ABF}$ & RGA & MS3D Otsu \\
\hline \multirow{4}{*}{ Slice \#022 } & 2 & 1806.9 & 1808.0 & 1808.8 & 1805.9 & 2257.5 \\
\hline & 3 & 2134.7 & 2149.4 & 2152.9 & 2119.6 & 2273.7 \\
\hline & 4 & 2260.3 & 2279.5 & 2284.0 & 2220.9 & 2357.9 \\
\hline & 5 & 2298.9 & 2311.1 & 2317.0 & 2258.5 & 2398.7 \\
\hline \multirow{4}{*}{ Slice \#032 } & 2 & 1805.7 & 1808.3 & 1809.3 & 1768.2 & 2591.6 \\
\hline & 3 & 2509.0 & 2547.3 & 2550.4 & 2490.0 & 2696.3 \\
\hline & 4 & 2638.2 & 2653.5 & 2656.0 & 2622.4 & 2717.8 \\
\hline & 5 & 2684.6 & 2697.3 & 2704.0 & 2661.3 & 2728.7 \\
\hline \multirow{4}{*}{ Slice \#042 } & 2 & 2115.5 & 2116.8 & 2704.0 & 2114.1 & 3027.7 \\
\hline & 3 & 2844.7 & 2882.8 & 2885.7 & 2777.0 & 3126.4 \\
\hline & 4 & 3107.5 & 3114.5 & 3117.6 & 3081.1 & 3180.1 \\
\hline & 5 & 3127.2 & 3153.3 & 3157.3 & 3096.6 & 3214.3 \\
\hline \multirow{4}{*}{ Slice \#052 } & 2 & 1568.4 & 1569.4 & 1569.4 & 1567.5 & 2841.7 \\
\hline & 3 & 2043.7 & 2090.78 & 2093.4 & 1987.6 & 2928.3 \\
\hline & 4 & 2468.6 & 2479.7 & 2482.5 & 2423.1 & 2969.0 \\
\hline & 5 & 2898.0 & 2928.6 & 2932.9 & 2853.3 & 2997.5 \\
\hline \multirow{4}{*}{ Slice \#062 } & 2 & 2156.8 & 2035.3 & 2158.6 & 2156.6 & 3351.6 \\
\hline & 3 & 2716.3 & 2765.9 & 2769.3 & 2607.7 & 3465.7 \\
\hline & 4 & 3190.5 & 3204.2 & 3206.1 & 3155.3 & 3517.1 \\
\hline & 5 & 3265.7 & 3304.9 & 3309.2 & 3212.7 & 3557.4 \\
\hline \multirow{4}{*}{ Slice \#072 } & 2 & 2081.9 & 2082.5 & 2082.9 & 2081.1 & 3187.1 \\
\hline & 3 & 2253.4 & 2260.7 & 2263.0 & 2213.2 & 3321.2 \\
\hline & 4 & 2377.8 & 2431.8 & 2434.0 & 2325.6 & 3385.2 \\
\hline & 5 & 3112.2 & 3123.7 & 3127.0 & 3073.9 & 3413.3 \\
\hline \multirow{4}{*}{ Slice \#082 } & 2 & 1696.2 & 1653.1 & 1653.4 & 1694.6 & 2925.0 \\
\hline & 3 & 1802.7 & 1815.1 & 1818.4 & 1768.2 & 3046.8 \\
\hline & 4 & 2038.9 & 2098.0 & 2100.0 & 1982.7 & 3099.7 \\
\hline & 5 & 2432.92 & 2499.2 & 2502.2 & 2367.9 & 3122.4 \\
\hline
\end{tabular}


Table 4 (continued)

\begin{tabular}{ccccccc}
\hline \multirow{2}{*}{$\begin{array}{c}\text { Testing } \\
\text { images }\end{array}$} & Number of & \multicolumn{5}{c}{ Objective values } \\
\cline { 3 - 7 } thresholds $(K-1)$ & PSO & BF & ABF & RGA & MS3D Otsu \\
\hline \multirow{2}{*}{ Slice \#092 } & 2 & 1567.8 & 1610.6 & 1612.5 & 1560.8 & $\mathbf{2 6 4 2 . 9}$ \\
& 3 & 1610.4 & 1656.6 & 1658.8 & 1567.0 & $\mathbf{2 7 0 5 . 4}$ \\
& 4 & 1906.4 & 1957.6 & 1961.8 & 1834.8 & $\mathbf{2 7 3 6 . 7}$ \\
& 5 & 1911.6 & 1975.6 & 1980.7 & 1847.0 & $\mathbf{2 7 5 6 . 8}$ \\
\hline \multirow{3}{*}{ Slice \#102 } & 2 & 1719.8 & 1730.8 & 1732.2 & 1682.7 & $\mathbf{2 5 6 0 . 2}$ \\
& 3 & 1820.1 & 1837.8 & 1843.0 & 1748.0 & $\mathbf{2 6 3 2 . 7}$ \\
& 4 & 1946.9 & 1987.1 & 1992.9 & 1896.7 & $\mathbf{2 6 5 5 . 1}$ \\
Slice \#112 & 5 & 2054.1 & 2108.9 & 2111.3 & 2003.1 & $\mathbf{2 6 7 3 . 2}$ \\
& 2 & 1837.9 & 1841.3 & 1843.8 & 1832.9 & $\mathbf{2 0 0 5 . 3}$ \\
& 3 & 1861.2 & 1893.2 & 1896.9 & 1833.1 & $\mathbf{2 0 8 1 . 6}$ \\
& 4 & 1954.9 & 1967.7 & 1971.2 & 1935.5 & $\mathbf{2 1 1 2 . 4}$ \\
\hline
\end{tabular}

Table 5 Comparison of the uniformity measure for different segmentation methods

\begin{tabular}{ccccccc}
\hline \multirow{2}{*}{$\begin{array}{c}\text { Testing } \\
\text { images }\end{array}$} & Number of & \multicolumn{5}{c}{ Uniformity measure $(u)$} \\
\cline { 3 - 7 } thresholds $(K-1)$ & PSO & BF & ABF & RGA & MS3D Otsu \\
\hline \multirow{2}{*}{ Slice \#022 } & 2 & 0.9552 & 0.9569 & 0.9569 & 0.9569 & $\mathbf{0 . 9 8 6 3}$ \\
& 3 & 0.9672 & 0.9708 & 0.9696 & 0.9769 & $\mathbf{0 . 9 8 1 0}$ \\
& 4 & 0.9420 & 0.9765 & 0.9698 & 0.9824 & $\mathbf{0 . 9 8 5 0}$ \\
Slice \#032 & 5 & 0.9435 & 0.9786 & 0.9785 & 0.9788 & $\mathbf{0 . 9 8 7 5}$ \\
& 2 & 0.9368 & 0.9342 & 0.9342 & 0.9342 & $\mathbf{0 . 9 8 5 1}$ \\
& 3 & 0.9619 & 0.9716 & 0.9600 & 0.9801 & $\mathbf{0 . 9 8 7 4}$ \\
& 5 & 0.9144 & 0.9697 & 0.9766 & 0.9848 & $\mathbf{0 . 9 8 5 8}$ \\
Slice \#042 & 5 & 0.9442 & 0.9668 & 0.9767 & $\mathbf{0 . 9 8 4 3}$ & 0.9839 \\
& 2 & 0.9271 & 0.9246 & 0.9246 & 0.9246 & $\mathbf{0 . 9 8 3 1}$ \\
& 3 & 0.9585 & 0.9721 & 0.9689 & 0.9548 & $\mathbf{0 . 9 8 3 8}$ \\
& 4 & 0.9465 & 0.9752 & 0.9821 & $\mathbf{0 . 9 8 6 5}$ & 0.9850 \\
\hline
\end{tabular}


Table 5 (contimued)

\begin{tabular}{|c|c|c|c|c|c|c|}
\hline \multirow{2}{*}{$\begin{array}{l}\text { Testing } \\
\text { images }\end{array}$} & \multirow{2}{*}{$\begin{array}{c}\text { Number of } \\
\text { thresholds }(K-1)\end{array}$} & \multicolumn{5}{|c|}{ Uniformity measure $(u)$} \\
\hline & & PSO & $\mathrm{BF}$ & $\mathrm{ABF}$ & RGA & MS3D Otsu \\
\hline \multirow{4}{*}{ Slice \#052 } & 2 & 0.9158 & 0.9128 & 0.9128 & 0.9128 & 0.9850 \\
\hline & 3 & 0.9523 & 0.9713 & 0.9673 & 0.9467 & 0.9855 \\
\hline & 4 & 0.9372 & 0.9764 & 0.9834 & 0.9856 & 0.9857 \\
\hline & 5 & 0.9240 & 0.9735 & 0.9782 & 0.9868 & 0.9865 \\
\hline \multirow{4}{*}{ Slice \#062 } & 2 & 0.9192 & 0.9047 & 0.9049 & 0.9015 & 0.9814 \\
\hline & 3 & 0.8777 & 0.9135 & 0.9029 & 0.9030 & 0.9826 \\
\hline & 4 & 0.9236 & 0.8856 & 0.8988 & 0.8989 & 0.9831 \\
\hline & 5 & 0.8508 & 0.9527 & 0.9325 & 0.9835 & 0.9851 \\
\hline \multirow{4}{*}{ Slice \#072 } & 2 & 0.9068 & 0.9041 & 0.9041 & 0.9041 & 0.9800 \\
\hline & 3 & 0.9034 & 0.9084 & 0.8985 & 0.8992 & 0.9824 \\
\hline & 4 & 0.8809 & 0.8876 & 0.8804 & 0.8666 & 0.9844 \\
\hline & 5 & 0.9531 & 0.8881 & 0.8876 & 0.9818 & 0.9848 \\
\hline \multirow{4}{*}{ Slice \#082 } & 2 & 0.9120 & 0.9091 & 0.9091 & 0.9091 & 0.9821 \\
\hline & 3 & 0.8852 & 0.8621 & 0.8661 & 0.8849 & 0.9843 \\
\hline & 4 & 0.8619 & 0.8479 & 0.8622 & 0.8695 & 0.9856 \\
\hline & 5 & 0.9372 & 0.9188 & 0.9105 & 0.9854 & 0.9855 \\
\hline \multirow{4}{*}{ Slice \#092 } & 2 & 0.9131 & 0.9156 & 0.9131 & 0.9131 & 0.9890 \\
\hline & 3 & 0.8607 & 0.8751 & 0.8827 & 0.8786 & 0.9893 \\
\hline & 4 & 0.9490 & 0.8583 & 0.8514 & 0.8641 & 0.9896 \\
\hline & 5 & 0.8684 & 0.8923 & 0.8401 & 0.9876 & 0.9901 \\
\hline \multirow{4}{*}{ Slice \#102 } & 2 & 0.9383 & 0.9250 & 0.9250 & 0.9250 & 0.9882 \\
\hline & 3 & 0.8768 & 0.8977 & 0.9097 & 0.9179 & 0.9892 \\
\hline & 4 & 0.9256 & 0.9410 & 0.9050 & 0.9871 & 0.9884 \\
\hline & 5 & 0.8446 & 0.9180 & 0.9181 & 0.9895 & 0.9884 \\
\hline \multirow{4}{*}{ Slice \#112 } & 2 & 0.9356 & 0.9403 & 0.9404 & 0.9404 & 0.9891 \\
\hline & 3 & 0.9147 & 0.9666 & 0.9769 & 0.9890 & 0.9910 \\
\hline & 4 & 0.9751 & 0.9824 & 0.9825 & 0.9896 & 0.9919 \\
\hline & 5 & 0.9735 & 0.9822 & 0.9830 & 0.9914 & 0.9919 \\
\hline
\end{tabular}

Summing up, uniformity measure and objective values produced by MS3D Otsu are better than those obtained by PSO, BF, ABF and RGA, which emphasizes the proposed method's best solution quality. MS3D Otsu preserves denoising performance of traditional 3D Otsu, while shows nice 
segmentation results. Therefore, the proposed method is superior enough for multilevel thresholding segmentation problem.

In our experiments, the proposed segmentation algorithm is also compared with other algorithms by medical diagnosis expertize in order to validate the performance of the proposed algorithm in real clinic diagnosis. In order to minimize the influence of personal preference, 10 observers from the Second Hospital of Jilin University participated in the evaluation program. To reduce the effect of testing environment, all the evaluations are conducted in a normal indoor office workspace. A customized MATLAB GUI was used to display images one set by one set on the screen. As shown in Fig.10, the original image is displayed in the first place, and seven segmented images at most are randomly displayed in the following place. In addition, the GUI provide zoom-up tool for observers to examine details. Each subject was asked to enter an integer score ranging from 1 to 5 according to their domain knowledge. After finishing one test set, subjects should submit their score and the system will display another set. The final subject evaluation result is the average of the scores.

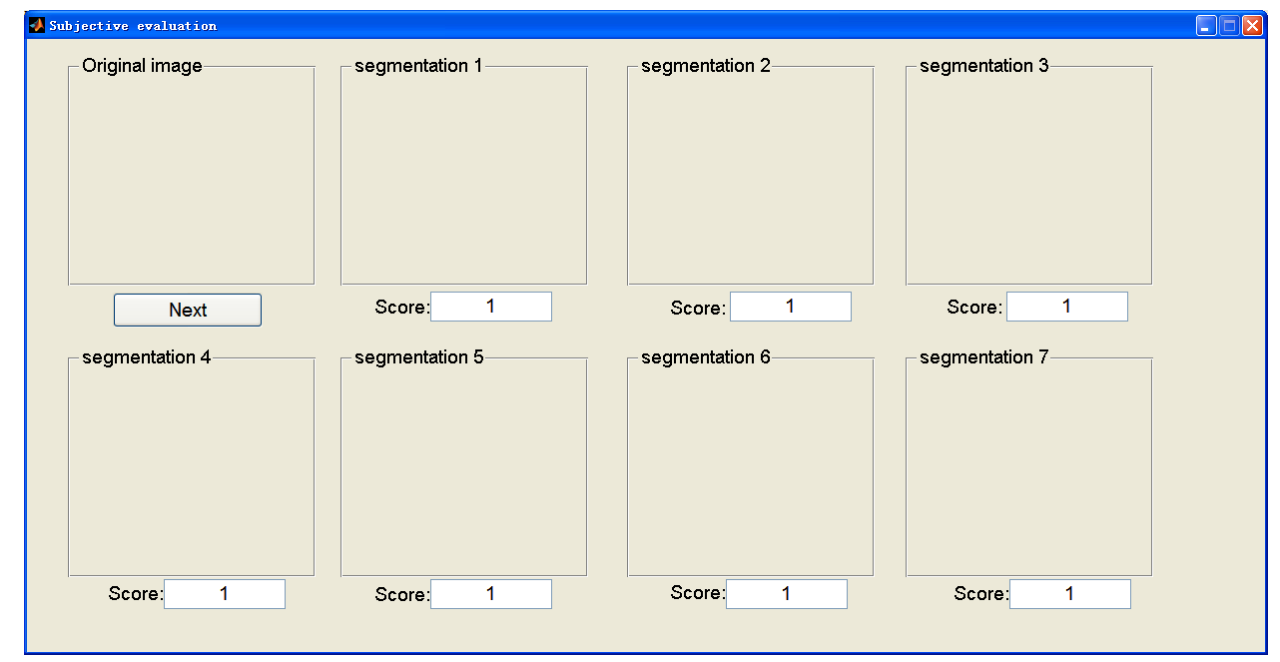

Fig.10 Matlab GUI for subjective evaluation of segmented images

Because of limited space, we listed averaged subjective score of each algorithm. As shown in Fig.11, it can be observed that the algorithm achieved the highest averaged score, which means that it is recognized by medical diagnosis experts.

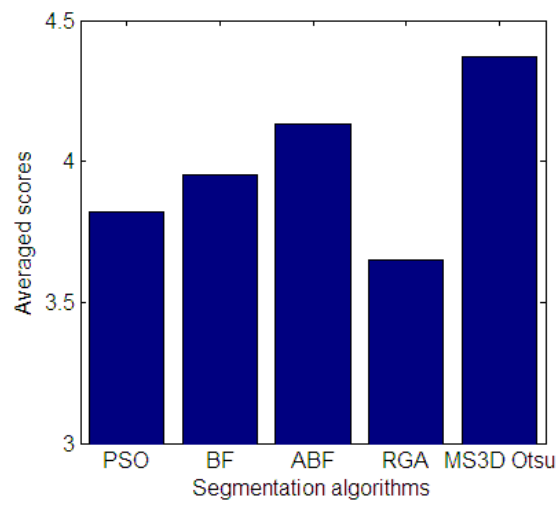

Fig.11 Averaged scores of different segmentation algorithms 


\subsection{Time complexity analysis}

The processing time of the proposed algorithm is mainly spent on two steps. First, the original image is segmented using the efficient 3D Otsu based on decomposition rule, where the time complexity is $O(L+L+L)=O(L)$. Second, the segmented image is filtered by the fast local Laplacian filtering, where the time complexity is $O\left(M^{*} N^{*} K\right)$. So, the total time complexity of the method is $O\left(L+M^{*} N^{*} K\right)$.

We test the proposed algorithm on the real images. All the experiments are implemented on a PC with an Intel(R) Xeon(R) CPU 3.10GHz and 3.24 GB RAM utilizing MATLAB 7.9.0 (R2009b). For $(K-1)=2,3,4,5$, the average running time of ten test images (slice \#022 \#112) by the proposed methods is listed in Fig.12.

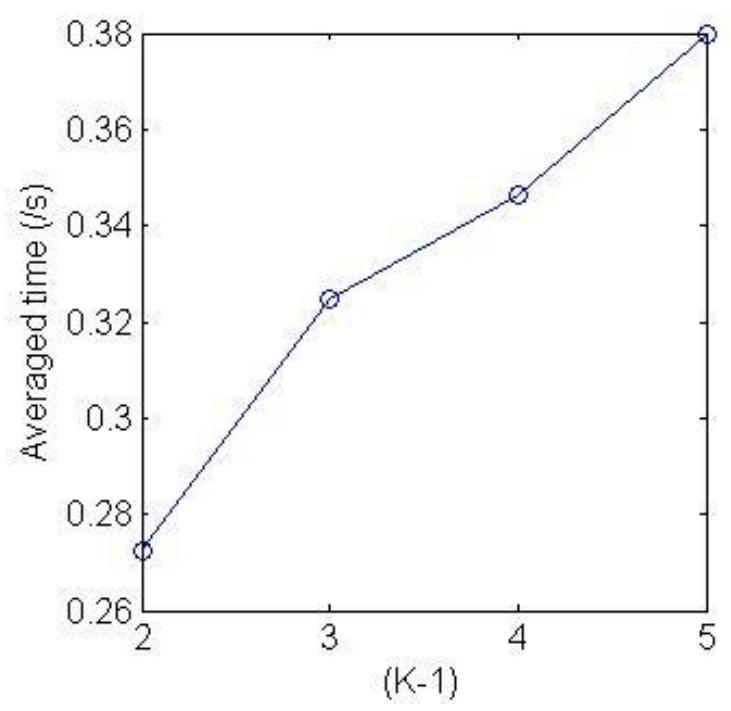

Fig.12 Averaged time consumption

From the figure, it can be observed that the average running time of the proposed method is lower than $0.5 \mathrm{~s}$ in the case of $(\mathrm{K}-1)=2,3,4$ and 5 . Hence, the algorithm can be integrated into real-time system.

\section{Conclusion}

In this paper, a new thresholding algorithm based on multi-scale 3D Otsu is proposed dedicated to medical images. The attractive features of the algorithm are that its segmentation results are stable and it is robust to noises. 3D Otsu can provide more precise segmentations comparing to 1D Otsu because of its capability of incorporating spatial information. In this paper, a variant of 3D Otsu based on dimension decomposition rule is invented in a bid to reduce time complexity without the loss of segmentation quality. The segmentation algorithm is designed as an iteration procedure. In each iteration, the image is segmented by the 3D Otsu, and then it is filtered by a fast local Laplacian filtering to get a smoothed image which will be input into the next iteration. Finally, the segmentation results are pooled to get a final segmentation using majority voting rules.

In the algorithm, there is one parameter, the image decomposition level scale, to be determined. We tested the algorithm on ten medical images with different scale, and the experimental results 
show that the optimal value of scale is 2 , which means that the best segmentation can be obtained by run once filtering and twice 3D Otsu. The proposed algorithm was compared with other state-of-art algorithms in the case of bi-level and multi-level thresholding. The results show that the proposed algorithm can generate more satisfactory segmentation maps from the aspect of observers. The proposed algorithm can be wildly used in clinical medicine. For example, it can automatically extract the region of interest (ROI), which is helpful to the analysis and recognition of medical image. It can also be used to measure the size or volume of an organ or tissue.

As the fusion rule is simple, the noises in original images cannot be perfectly dealt with. Hence, designing a more sophisticated fusion rule is one of our research directions. In addition, we will further research on reducing time complexity of the algorithm.

\section{Acknowledgements}

The authors would like to express our gratitude to the editors and anonymous reviewers for their comments and suggestions. The work was supported by Projects in the National Science \& Technology Pillar Program, China (2012BAH48F02), National Science Foundation of China (Grant No.61272209), Technology Development Plan of Jilin Province (201105017), and Agreement of Science \& Technology Development Project, Jilin Province (No.20150101014JC). We would also like to thank http://www.med.harvard.edu/aanlib/home.html for providing us the medical images.

\section{References}

[1] L. Zhang, Q. Ji, A Bayesian network model for automatic and interactive image segmentation, IEEE Transactions on Image Processing 20(9) ( 2011) 2582-2593.

[2] L. Tlig, M. Sayadi, F. Fnaiech, A new fuzzy segmentation approach based on S-FCM type 2 using LBP-GCO features, Signal Processing: Image Communication, 27(6), (2012) 694-708.

[3] S. Sarkar, S. Das, S.S. Chaudhuri, A multilevel color image thresholding scheme based on minimum cross entropy and differential evolution, Pattern Recognition Letters 54 (2015) 27-35.

[4] D. Gómez, J. Yáñez, C. Guada, et al., Fuzzy image segmentation based upon hierarchical clustering, Knowledge-Based Systems 87 (2015) 26-37.

[5] X.J. Jing, J.F. Li, Y.L. Liu, Image Segmentation Based on 3-D Maximum Between-Cluster Variance, Acta Electronica Sinica 31(9) (2003) 1281-1285.

[6] Q. Miao, P. Xu, T. Liu, et al., A Novel Fast Image Segmentation Algorithm for Large Topographic Maps, Neurocomputing 168 (2015) 808-822.

[7] A.K. Bhandari, A. Kumar, G.K. Singh, Tsallis Entropy based Multilevel Thresholding for Colored Satellite Image Segmentation using Evolutionary Algorithms, Expert Systems with Applications 42(22) (2015) 8707-8730.

[8] A.K. Bhandari, A. Kumar, G.K. Singh, Modified artificial bee colony based computationally efficient multilevel thresholding for satellite image segmentation using Kapur's, Otsu and Tsallis functions, Expert Systems with Applications 42(3) (2015) 1573-1601. 
[9] C. Li, X. Wang, S. Eberl, et al., Supervised Variational Model With Statistical Inference and Its Application in Medical Image Segmentation, IEEE Transactions on Biomedical Engineering 62(1) (2015) 196-207.

[10] E. Smistad, T.L. Falch, M. Bozorgi, et al., Medical image segmentation on GPUs - A comprehensive review, Medical Image Analysis 20 (2015) 1-18.

[11] S, Aja-Fernández, A.H. Curiale, G. Vegas-Sánchez-Ferrero, A local fuzzy thresholding methodology for multiregion image segmentation, Knowledge-Based Systems 83 (2015) 1-12.

[12] S. Kobashi, M. Matsui, N. Inoue, K. Kondo, T. Sawada, Y. Hata, Cerebral Cortex Segmentation with Adaptive Fuzzy Spatial Modeling in 3.0T IR-FSPGR MR Images, Journal of Japan Society for Fuzzy Theory \& Intelligent Informatics 20(1) (2008) 29-40.

[13] K.R. Hahn, K. Rodenacker, D.P. Auer, Segmentation of the human cerebral cortex for advanced morphometric analysis, Neuroimage 11(5) (2000) S500-S500.

[14] Y. Guo, A. Şengür, J.W. Tian, A Novel Breast Ultrasound Image Segmentation Algorithm Based on Neutrosophic Similarity Score and Level Set. Computer Methods and Programs in Biomedicine, 2015 (available online).

[15] C. Li, J.C. Gore, C. Davatzikos, Multiplicative intrinsic component optimization (MICO) for MRI bias field estimation and tissue segmentation, Magnetic resonance imaging 32(7) (2014) 913-923.

[16] S. Manikandan, K. Ramar, M.W. Iruthayarajan, et al., Multilevel thresholding for segmentation of medical brain images using real coded genetic algorithm, Measurement 47 (2014) 558-568.

[17] P.D. Sathya, R. Kayalvizhi, Optimal segmentation of brain MRI based on adaptive bacterial foraging algorithm, Neurocomputing 74(14) (2011) 2299-2313.

[18] M. Maitra, A. Chatterjee, A novel technique for multilevel optimal magnetic resonance brain image thresholding using bacterial foraging, Measurement 41(10) (2008) 1124-1134.

[19] D. Gómez, J. Yáñez, C. Guada, et al., Fuzzy image segmentation based upon hierarchical clustering, Knowledge-Based Systems 87 (2015) 26-37.

[20] C. Li, R. Huang, Z. Ding, et al., A level set method for image segmentation in the presence of intensity in homogeneities with application to MRI, IEEE Transactions on Image Processing 20(7) (2011) 2007-2016.

[21] Dai S, Lu K, Dong J, et al. A novel approach of lung segmentation on chest CT images using graph cuts. Neurocomputing 168 (2015) 799-807.

[22] Otsu N. A threshold selection method from gray-level histograms. Automatica 11(285-296) (1975) 23-27.

[23] J. Kittler, J. Illingworth, Minimum error thresholding, Pattern recognition 19(1) (1986) 41-47.

[24] J.N. Kapur, P.K. Sahoo, A.K.C. Wong, A new method for gray-level picture thresholding using the entropy of the histogram, Computer vision, graphics, and image processing 29(3) 
(1985) 273-285.

[25] J. Poljak, G. Botella, C. Garcia, S.M. Poljacek, M. Prieto-Matias, F. Tirado, Offset Printing Plate Quality Sensor on a Low-Cost Processor, Sensors 13(11) (2013) 14277-14300.

[26] L.Y. Duan, R. Ji, Z. Chen, T. Huang, Towards Mobile Document Image Retrieval for Digital Library, IEEE Transactions on Multimedia 16(2) (2014) 346-359.

[27] Carlos García Sánchez, Guillermo Botella Juan, Fermín Ayuso Márquez, Diego González Rodríguez, Manuel Prieto-Matías, Francisco Tirado Fernández. Implementation of a Low-Cost Mobile Devices to Support Medical Diagnosis. Computational \& Mathematical Methods in Medicine,(4) (2013) 287089-287089.

[28] J. Zhao, B. Xie, X. Huang, Real-time lane departure and front collision warning system on an FPGA, High Performance Extreme Computing Conference (HPEC), (2014) 1 - 5.

[29] Q. Lin, C. Ou, Tsallis entropy and the long-range correlation in image thresholding, Signal Processing 92(12) (2012) 2931-2939.

[30] H. Cai, Z. Yang, X. Cao, et al., A new iterative triclass thresholding technique in image segmentation, IEEE Transactions on Image Processing 23(3) (2014) 1038-1046.

[31] J.W. Long, X.J. Shen, H.P. Chen, Adaptive minimum error thresholding algorithm, Acta Automatica Sinica 7 (2012) 1134-1144.

[32] S. Dey, I. Saha, S. Bhattacharyya, et al., Multi-level thresholding using quantum inspired meta-heuristics, Knowledge-Based Systems 67 (2014) 373-400.

[33] K. Hammouche, M. Diaf, P. Siarry, A multilevel automatic thresholding method based on a genetic algorithm for a fast image segmentation, Computer Vision \& Image Understanding 109(2) (2008) 163-175.

[34] X. Chen, J.K. Udupa, U. Bagci, et al., Medical image segmentation by combining graph cuts and oriented active appearance models, IEEE Transactions on Image Processing 21(4) (2012) 2035-2046.

[35] J. Jin, L. Yang, X. Zhang, M. Ding, Vascular tree segmentation in medical images using Hessian-based multiscale filtering and level set method, Computational \& Mathematical Methods in Medicine 2013 (2013) 502013(1-9).

[36] S. Paris, S.W. Hasinoff, J. Kautz, Local Laplacian filters: edge-aware image processing with a Laplacian pyramid, ACM Trans. Graph. 30(4) (2011) 68.

[37] M. Aubry, S. Paris, S.W. Hasinoff, et al., Fast Local Laplacian Filters: Theory and Applications, ACM Trans. Graph. 33(5) (2014) 1935-1946.

[38] P. Sthitpattanapongsa, T. Srinark, An Equivalent 3D Otsu's Thresholding Method, In Advances in Image and Video Technology: 5th Pacific Rim Symposium, Gwangju, South Korea, (2011) 358-369.

[39] X.J. Shen, J.W. Long, H.P. Chen, et al., Otsu thresholding algorithm based on rebuilding and dimension reduction of the 3-dimensional histogram, Acta Electronica Sinica 39(5) (2011) 
1108-1114.

[40] A. Chander, A. Chatterjee, P. Siarry, A new social and momentum component adaptive PSO algorithm for image segmentation, Expert Systems with Applications 38(5) (2011) 4998-5004. 